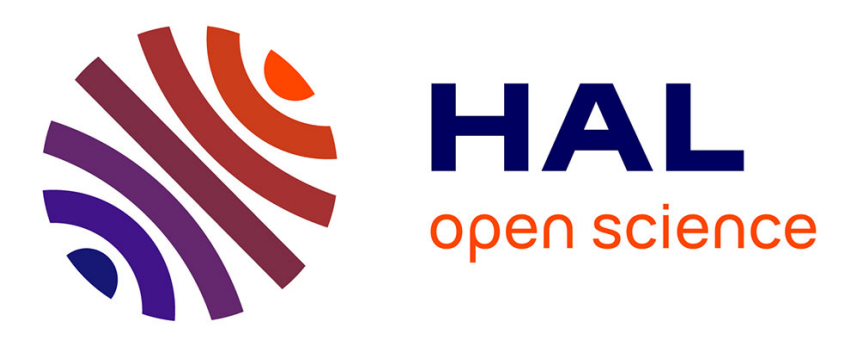

\title{
Aeroacoustic Design and Broadband Noise Predictions of a Fan Stage with Serrated Outlet Guide Vanes
} Cyril Polacsek, Adil Cader, Martin Buszyk, Raphaël Barrier, Fernando Gea-Aguilera, Hélène Posson

\section{- To cite this version:}

Cyril Polacsek, Adil Cader, Martin Buszyk, Raphaël Barrier, Fernando Gea-Aguilera, et al.. Aeroacoustic Design and Broadband Noise Predictions of a Fan Stage with Serrated Outlet Guide Vanes. Physics of Fluids, 2020, 32 (10), 10.1063/5.0020190 . hal-02968328v2

\section{HAL Id: hal-02968328 \\ https://hal.science/hal-02968328v2}

Submitted on 16 Dec 2020

HAL is a multi-disciplinary open access archive for the deposit and dissemination of scientific research documents, whether they are published or not. The documents may come from teaching and research institutions in France or abroad, or from public or private research centers.
L'archive ouverte pluridisciplinaire HAL, est destinée au dépôt et à la diffusion de documents scientifiques de niveau recherche, publiés ou non, émanant des établissements d'enseignement et de recherche français ou étrangers, des laboratoires publics ou privés. 


\title{
Aeroacoustic Design and Broadband Noise Predictions of a Fan Stage with Serrated Outlet Guide Vanes
}

\author{
C. Polacsek, ${ }^{1, a}$ A. Cader, ${ }^{1, b}$ M. Buszyk, ${ }^{1, b}$ R. Barrier, ${ }^{2, c}$ F. Gea-Aguilera, ${ }^{3, d}$ and \\ H. Posson ${ }^{3, d}$ \\ ${ }^{1}$ ONERA -The French Aerospace Lab, Numerical Aeroacoustics Unit, F92322, Châtillon, France \\ ${ }^{2}$ ONERA -The French Aerospace Lab, Turbomachinery Applied Aerodynamics Unit, F92190, Meudon, France \\ ${ }^{3}$ Safran Aircraft Engines, Moissy-Cramayel, 77550, France
}

This work, realized in the framework of the European project TurboNoiseBB, presents an advanced aeroacoustic design methodology of Outlet Guide Vanes (OGVs) with leading edge serrations, including details on their broadband noise and aerodynamic performance. The serrated OGV corresponds to a modified stator from an aero-engine fan stage tested at the AneCom Aerotest's facility (Germany). Sinusoidal leading edge patterns with varying amplitude and wavelength along the span are designed in collaboration with Safran Aircraft Engines. Serrations are adjusted to account for the turbulence characteristics provided by Reynolds-Averaged Navier-Stokes (RANS) calculations. Optimal parameters are found using simple design rules discussed in the paper. Down selection of serrated OGV designs (patent pending) are conducted through a RANS control of aerodynamic performances and in accordance with industrial specifications, ensuring acceptable penalties on the loss coefficient and isentropic efficiency. Broadband noise simulations are performed using a computational aeroacoustic (CAA) code that solves the linearized Euler equations with a synthetic turbulence model. Additionally, the acoustic response of the serrated leading edge airfoils is also estimated using the most relevant analytical formulation in the literature, based on the Wiener-Hopf technique. Numerical predictions at approach conditions are compared to available experimental measurements (on the untreated baseline case) and analytical Amiet-based and Wiener-Hopf solutions, showing a satisfactory agreement in the sound power spectrum in the bypass duct. Finally, the acoustic performances estimated at the design stage are numerically assessed by both $\mathrm{CAA}$ and Wiener-Hopf methods, providing around $2.5 \mathrm{~dB}$ and $3.5 \mathrm{~dB}$ reduction on the overall power level, respectively.

\section{INTRODUCTION}

In the past ten years, turbulence interaction noise reduction using leading edge (LE) serrations has been extensively studied. ${ }^{1-10}$. One of the first studies related to the design and manufacture of airfoils with LE serrations was performed by ONERA (Refs. ${ }^{1-2}$ ) to reduce broadband noise from Outlet Guide Vanes (OGVs) in turbofan engines, as part of the FLOCON (Ref. ${ }^{11}$ ) European project.

\footnotetext{
a) Senior Aeroacoustics Engineer, Department of Aerodynamics, Aeroelasticity and Acoustics. Author to whom correspondence should be addressed. Electronic mail: cyril.polacsek@onera.fr.

b) $\mathrm{Ph}$. D. Student, Department of Aerodynamics, Aeroelasticity and Acoustics.

c) Research Aerodynamics Engineer, Department of Aerodynamics, Aeroelasticity and Acoustics.

d) R\&D Aeroacoustics Engineer, Aerodynamics and Acoustics Department.
} 
Oldest studies on LE serrations initiated in the seventies by Hersh et al. ${ }^{12}$, as well as more recent investigations at the time of FLOCON project, only focused on flow induced noise (i.e. airfoil self-noise) on fixed airfoils ${ }^{13}$ or rotating blades ${ }^{14}$. Although the airfoil self-noise may be relevant, this is a quite different source generation mechanism compared to the turbulence-airfoil interaction, considered to be the main contributor to the broadband noise emission from the fan stage of modern aeroengines $^{15}$. Recently, several institutes and authors in Europe have realized fruitful studies, with in particular noteworthy publications from ISVR $^{4-6}$ and University of Cambridge ${ }^{8}$. Although promising results have been found in experimental ${ }^{2-5}$, analytical $^{8}$, and numerical ${ }^{2,6}$ works using isolated airfoils, the benefit of leading edge serrations on realistic rotor/stator stages $^{7,9,10}$ remains quite challenging to assess from an industrial perspective.

The present work, performed in the framework of the European project TurboNoiseBB, presents the aeroacoustic design of leading edge serrations applied to a realistic fan stage from a turbofan engine at a reduced scale, including details on their broadband noise and aerodynamic performance. The investigated fan-OGV stage has been recently tested at AneCom Aerotest's facility ${ }^{16}$ (Germany) and shared with the Consortium partners. As experimental data were not available at the beginning of this work, the design methodology is fully based on turbulence characteristics from Reynolds-Averaged NavierStokes (RANS) calculations performed by ONERA ${ }^{17}$ and DLR. ${ }^{19}$ Broadband noise predictions are assessed using an in-house computational aeroacoustic (CAA) code that solves the linearized Euler equations with a synthetic turbulence method. ONERA's hybrid methodology and first simulations on the baseline case have already been presented by Cader et al. ${ }^{17} \mathrm{~A}$ similar method is used here to assess the acoustic performance of an optimized low-noise OGV concept, which extends preliminary results reported in $2019 .^{10}$ RANS calculations provide the turbulence characteristics of turbulent wakes interacting with the stator. This information is necessary to adjust the serration geometry (wavelength and amplitude of sinusoidal LE) and to calibrate the isotropic turbulence spectrum model that is used for the 3D CAA simulation. Fan-OGV simulations using a mixing plane approach are analyzed to assess the aerodynamic behavior of several serrated OGVs in comparison to the baseline case (with straight leading edge). Thus, an optimized design for a low-noise OGV can be identified from the assessment of the aerodynamic properties from RANS calculations and the noise reduction from CAA. In addition to the numerical predictions, a semi-analytical approach based on a the Wiener-Hopf (WH) formulation developed by Ayton and $\mathrm{Kim}^{8}$ is used to estimate the acoustic response of the most promising OGV design. Although the model assumes two-dimensional far-field radiation and a semi-infinite flat plate, a finite span correction is introduced here and the modeil is adapted to account for radial variations of the serration geometry along the span using a strip theory approach. Analytical WH predictions are validated using Amiet's model ${ }^{28}$ for an airfoil with straight leading edge, and available numerical and experimental results from a serrated LE airfoil studied by ONERA [Refs. $\left.{ }^{1-2}\right]$. Then, sound power level (PWL) 
reductions assessed from the most promising low-noise OGV using the analytical model are discussed and compared to the CAA results.

The paper is organized as follows:

- Section II discusses recent works in the field and design rules to choose relevant LE parameters for broadband noise reduction on isolated airfoils;

- $\quad$ Section III briefly presents the fan stage and experimental set-up;

- $\quad$ Section IV describes the design methodology for serrated OGVs using RANS information;

- Section V is devoted to the control of aerodynamic performances with the down selection of low-noise OGVs;

- Section VI presents the CAA method and numerical noise predictions on the baseline case (for which a comparison to AneCom measurements is proposed) and a low-noise OGV;

- Section VII, presents the WH model with suggested adaptations which are applied to FLOCON and TurboNoiseBB configurations;

- $\quad$ Finally, the main findings of this paper and future work topics are given in Section VIII.

\section{BACKGROUND ON ACOUSTIC PERFORMANCES OF LEADING EDGE SERRATIONS}

In the last decade, several works have studied the effects of leading edge serrations on noise. Some of the most relevant results in the field have been obtained from experimental investigations performed by ISVR on isolated airfoils. Based on previous publications from ONERA ${ }^{2}$ and ISVR, ${ }^{4-5}$ one can identify two major noise reduction mechanisms in the case of sinusoidal wavy patterns, as shown in Fig. 1. These noise reduction mechanisms are listed below:

- A reduction in the correlation between adjacent noise sources along the span, which is related to the transverse correlation length scale $\Lambda_{t}$, and therefore to the turbulence integral length scale, $\Lambda$;

- A modification of the aeroacoustic response giving rise to a loss of efficiency in the acoustic radiation from incoming gusts. Clair et al. $^{2}$ proposed an explanation based on a simple reformulation of the dispersion relation in the presence of sinusoidal serrations (approximated by a sawtooth pattern), which shows a filtering of the incoming parallel gusts when the

inclination angle of the serrations is close to $90^{\circ}$. This angle, $\theta_{s}$, is defined by $\theta_{s}=\tan ^{-1}\left(\frac{4 h_{s}}{\lambda_{s}}\right)$, which highlights the role 
of the serration amplitude, $h_{s}$, and wavelength, $\lambda_{s}$, in the noise reduction mechanism. This finding has been confirmed by recent experimental ${ }^{4-5}$ and analytical studies ${ }^{8}$.

Based on the above observations, the best compromise for isotropic turbulence is to set the serration wavelength to $\lambda_{s}=$ $4 \Lambda_{t}=2 \Lambda$. Another key parameter is the choice of a convenient ratio $h_{s} / \lambda_{s}$. Although the acoustic benefit could be improved by increasing $h_{s}$, a practical limitation is required to avoid a noticeable loss of aerodynamic performance for industrial applications, as discussed by Reboul et al. ${ }^{7}$ Hence, setting $h_{s} / \lambda_{s}=1$ gives $\theta_{s}=76^{\circ}$, which can be considered as a reasonable value close to the optimum design according to Narayanan et al. ${ }^{4}$ Another important result highlighted by Chaitanya et al. ${ }^{5}$ is that the sound power attenuation, $\triangle P W L$, that can be achieved by a single-wavelength serrated LE, is approximately proportional to the Strouhal number, $S_{t h}=f h_{s} / U$, at the optimum serration wavelength, where $U$ is the freestream velocity and $f$ is the frequency. The following empirical law was proposed:

$$
\Delta P W L(d B)=10+10 \log _{10}\left(S_{t h}\right)
$$

In practice, a significant PWL reduction $(>3 \mathrm{~dB})$ can be expected if $S_{t h} \geq 0.2$ for isolated airfoils with leading edge serrations. Such a criterion is used in Section IV for a preliminary assessment of the basic design parameters.

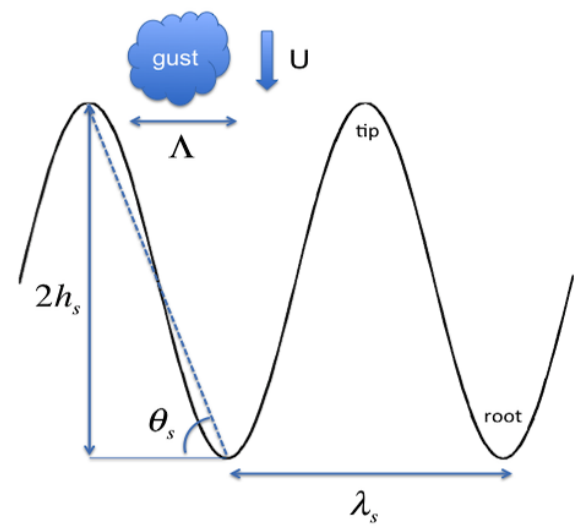

FIG. 1. Sketch of sinusoidal LE serrations with main relevant design parameters

\section{FAN STAGE AND EXPERIMENTAL SET-UP}

The AneCom AeroTest Rotor1 (ACAT1) model used in this study is a $85-\mathrm{cm}$ diameter rotor with a fan-OGV stage equipped with 20 blades and 44 vanes (Fig. 2, top). The acoustic calculations are performed at approach (APP) operating point (OP), while the aerodynamic performances are investigated in priority at the aerodynamic design point (ADP). The aerodynamic and acoustic databases were obtained in a test campaign ${ }^{19-20}$ conducted in 2018 at the Universal Fan Facility for 
Acoustics (UFFA), see Fig. 2 (bottom). The present investigation is limited to the baseline configuration with a rotor-stator spacing that is approximately equal to 1.5 times the rotor axial chord length, $c$. Hot-wire probes were used to measure the statistics of the turbulent wakes in the interstage (Fig. 2, top), and to validate the CFD solutions at this position. Acoustic measurements were obtained by using different microphone arrays (Fig. 2, bottom), aiming to assess the forward far-field radiation (with a free-field microphone array) and the in-duct sound field, with circumferential (CMD1 and CMD3) and axial (AX1) wall-flush mounted microphone arrays. Experimental BBN spectra were achieved by DLR using advanced signal processing techniques ${ }^{19-20}$. Sound power spectra reported in this paper were obtained from a wavenumber decomposition technique to remove the hydrodynamic fluctuations, which is necessary to capture the sound pressure field, using a duct mode analysis with an equal energy density distribution assumption. Good repeatability of raw acoustic data acquired during the tests was observed, so that main experimental uncertainties should be attributed to the post-processing assumptions to assess the in-duct sound power. PWL deviations that are within $\pm 2 \mathrm{~dB}$ can be expected.

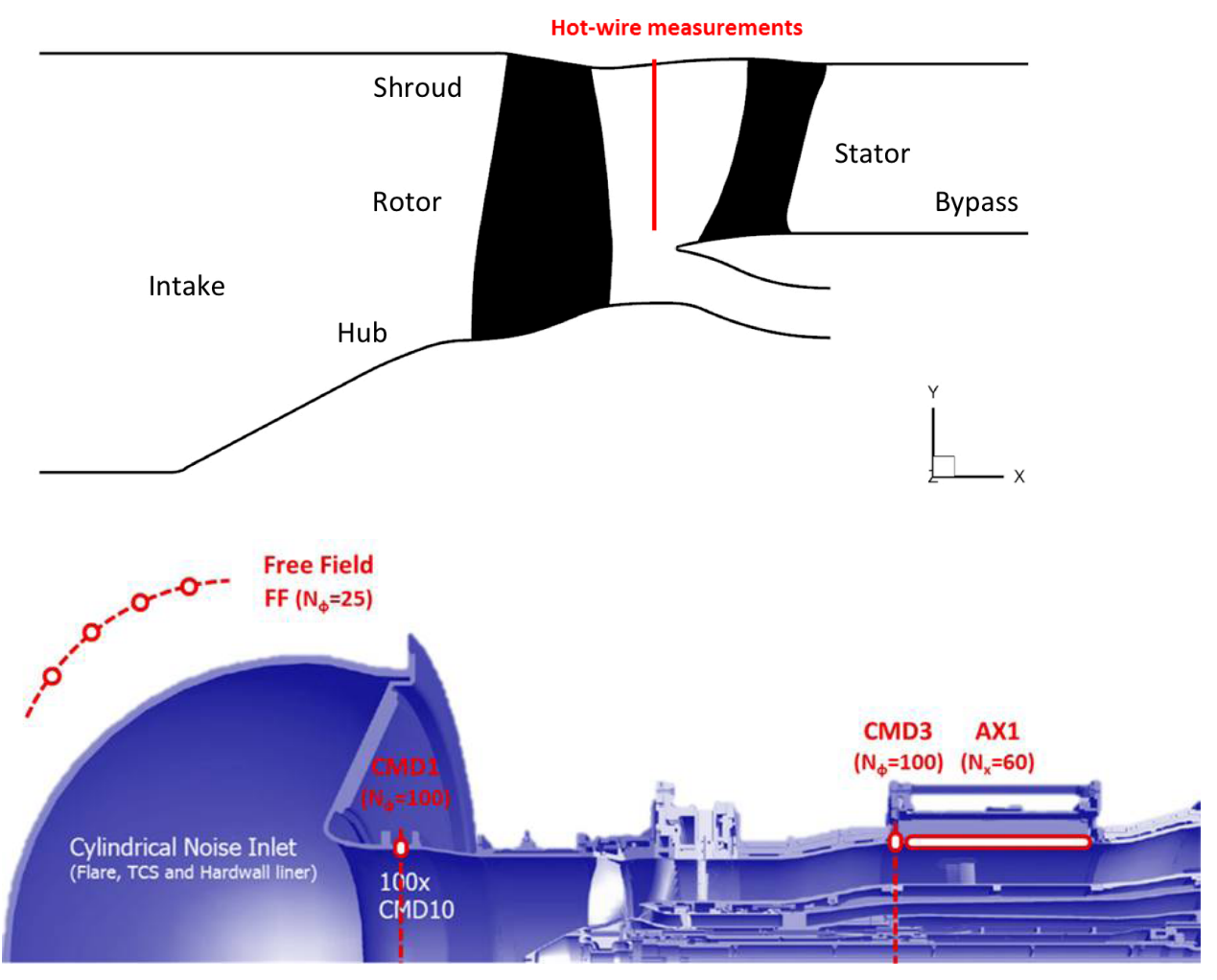

FIG. 2. ACAT1 fan stage geometry (top) and sketch of the rig set-up at AneCom's UFFA (bottom, reprint with TurboNoiseBB Consortium permission). 


\section{LOW-NOISE OGV DESIGN USING RANS DERIVED INFORMATION}

In this study, RANS simulations are based on $k-\ell$ Smith and $k-\omega$ Menter SST turbulence models from ONERA and

DLR using $e l s A^{21}$ and TRACE codes, respectively. ${ }^{22}$ For fast assessment of major aerodynamic features of a turbofan stage, a mixing plane approach is currently adopted in fan-OGV calculations instead of expensive unsteady RANS simulations. Basically, the CFD variables are circumferentially averaged over a cross-section (mixing plane) located at the interface between the rotating fan and fixed OGV domains. Thus, the mixing plane is used as a suited boundary condition to exchange the steady data fields across the interface. The mixing plane location is visible in Fig. 3, left (see also Fig. 13 in Section VI.B). As discussed in Section VI, RANS mixing plane calculations are required to provide the mean flow used by the CAA, whereas fan wake characteristics can be obtained from a fan alone steady RANS calculation by extending the mesh up to the OGV region (Fig. 3, right). For most practical applications, RANS wake characteristics from fan-OGV simulations can be only extracted right upstream of the mixing plane (Fig. 3, left), as the wakes are damped by the mixing process a few cells behind, whereas RANS outputs from fan alone simulations (Fig. 3, right) are extracted close to the LE of the OGV. Relevant parameters for the rotor-stator interaction noise are the circumferentially-averaged profiles along the span for the turbulence intensity (TI), the turbulence length scale (TLS or $\Lambda$ ), and the absolute (streamwise) mean flow velocity. These data are also used as inputs to an in-house acoustic code based on Amiet's theory ${ }^{28}$ that has been extended to account for a ducted annular cascade. This code can be used to estimate the in-duct PWL spectra, as discussed in Section VII. Two approaches are considered to estimate the TLS profiles. The first approach, which is referred to as "Pope", relates $\Lambda$ to the RANS turbulence model variables ${ }^{23}$ :

$$
\Lambda=\frac{C_{\mathrm{Re}}}{C_{\mu}} \frac{k^{1 / 2}}{\omega} \approx \frac{C_{\mathrm{Re}}}{C_{\mu}^{3 / 4}} \ell
$$

In Eq. (2), $k$ is the turbulent kinetic energy, $\omega$ is the specific dissipate rate, $C_{\mu}=0.09$, and $C_{R e}$ is set equal to 0.45 , according to Pope's work. ${ }^{24}$ The second approach, which is referred to as "Ganz", uses an empirical Gaussian wake approximation proposed by Ganz et al. ${ }^{25}$, that relates $\Lambda$ to the Gaussian wake width $\left(L_{w}\right)$ as $\Lambda=0.21 L_{w}$. As discussed in Ref. ${ }^{22}$, the choice of the RANS turbulence model as well as the way adopted to assess the TLS can have a significant impact on the noise results. This is the reason why two major models ( $k-\ell$ Smith and $k-\omega$ Menter SST) that are often used for turbomachinery applications have been selected. This is to account for variations from CFD profiles in the design process, at approach OP. TLS profiles from RANS ONERA simulations are plotted in Fig. 4. Depending on the RANS turbulence model and the approach used for the TLS calculation, the circumferentially-averaged profiles show different trends in the radial direction, 
particularly in the fan tip region. DLR RANS post-processed data (TI and TLS profiles), obtained at three OP conditions, approach (APP), cutback (CUTB), sideline (SDL), are plotted in Fig. 5. Results show dominant turbulence levels in the casing region and relatively similar TLS profiles for the three flow conditions.
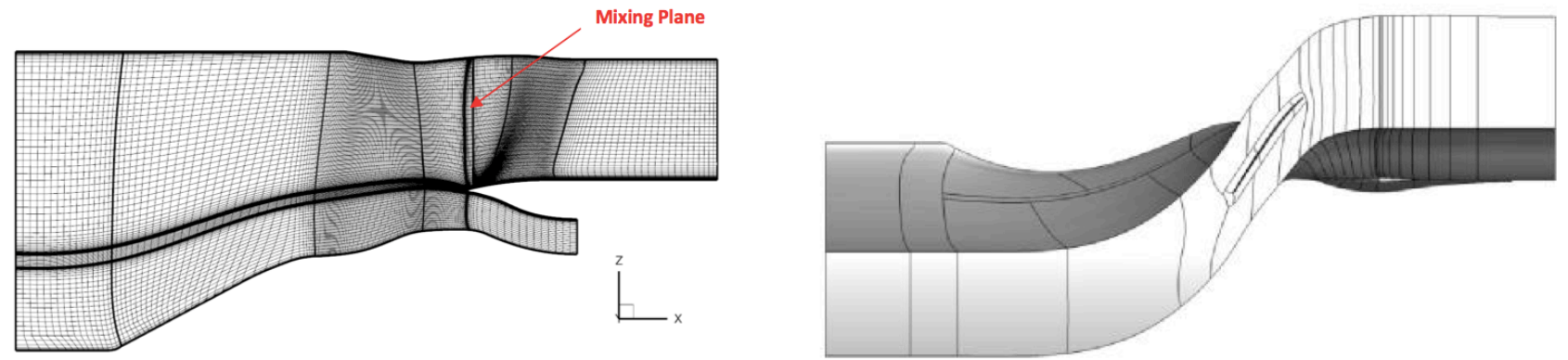

FIG. 3. Cross-section of the fan-OGV grid with a mixing plane (left) and fan alone domain (right) used for ONERA RANS calculations.

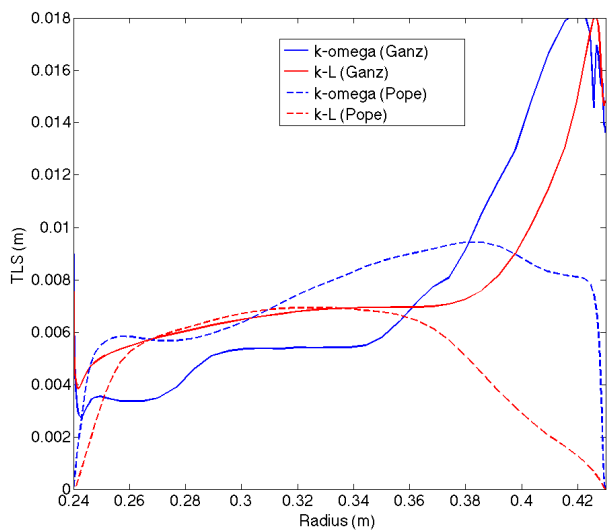

FIG. 4. Radial profiles of circumferentially-averaged TLS from fan alone RANS simulations at approach OP performed by ONERA with $k-\ell$ and $k-\omega$ turbulence models.
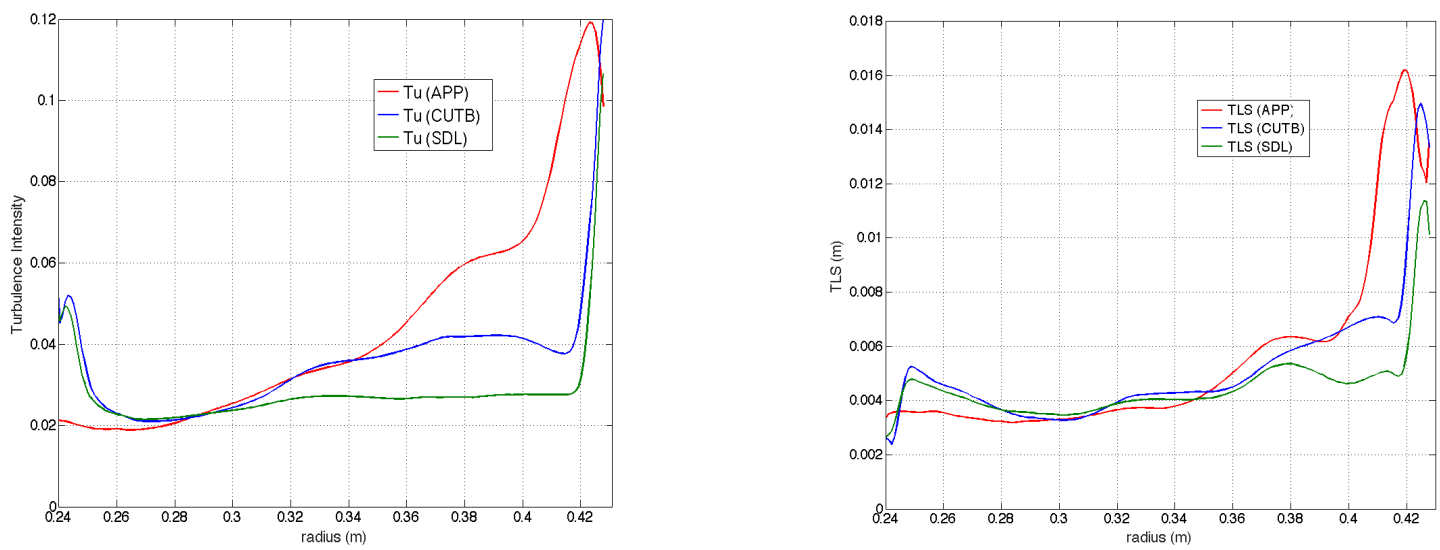

FIG. 5. Radial profiles of circumferentially-averaged TI (left) and Ganz-based TLS (right) from fan-OGV RANS simulations performed by DLR using $k-\omega$ turbulence model at approach, cutback and sideline OPs.

Initial serrated OGV designs have been conceived by using the averaged TLS values in the radial direction from Fig. 6. Design rules described in Section II led to 12-waves "GanzUniform" and 20-waves "PopeUniform" serrated OGVs designs, which are shown in Fig. 7. 2D drawings using the flat plate approximation (Fig. 7, left) are rebuilt in 3D (Fig. 7, right) using 
an in-house modeler, ersatZ. This code applies a contouring process from the leading edge, while preserving the mean camber line. It can be inferred from Figs. 6 and 7 that the serrated LE of the OGV is significantly influenced by the TLS definition from a RANS simulation, which may vary the amplitude and wavelength of the optimized design. Table I shows an estimation of the potential noise reduction for the "GanzUniform" OGV design by using Eq. (1), where the Strouhal number is based on $h_{s}$ and the peak frequency, $f_{\text {peak }}$, which corresponds to the frequency at which the broadband noise spectrum is at a maximum for the various OP, as discussed in Section VII. This preliminary assessment, which is valid for isolated airfoils in isotropic turbulence, indicates that a PWL reduction in broadband noise of at least $4 \mathrm{~dB}$ could be achieved from an OGV with optimized LE serrations.

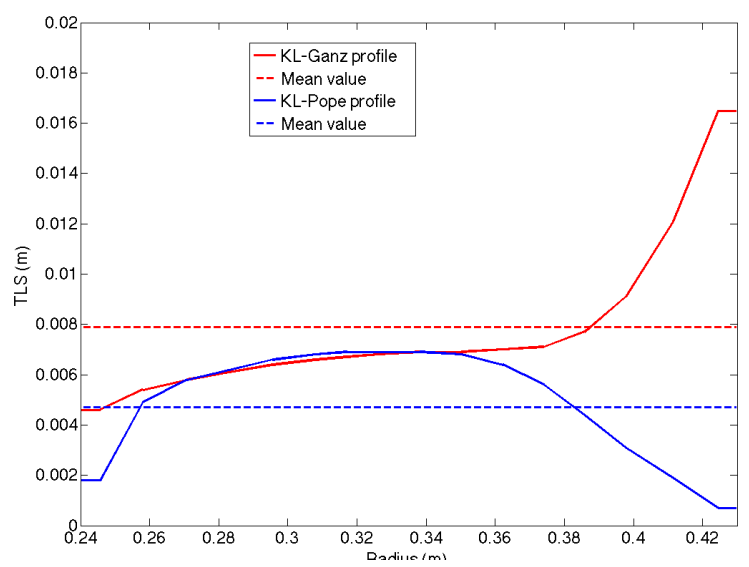

FIG. 6. Averaged TLS profile in the radial direction from $k-\ell$ turbulence model, obtained using data from Fig. 4.
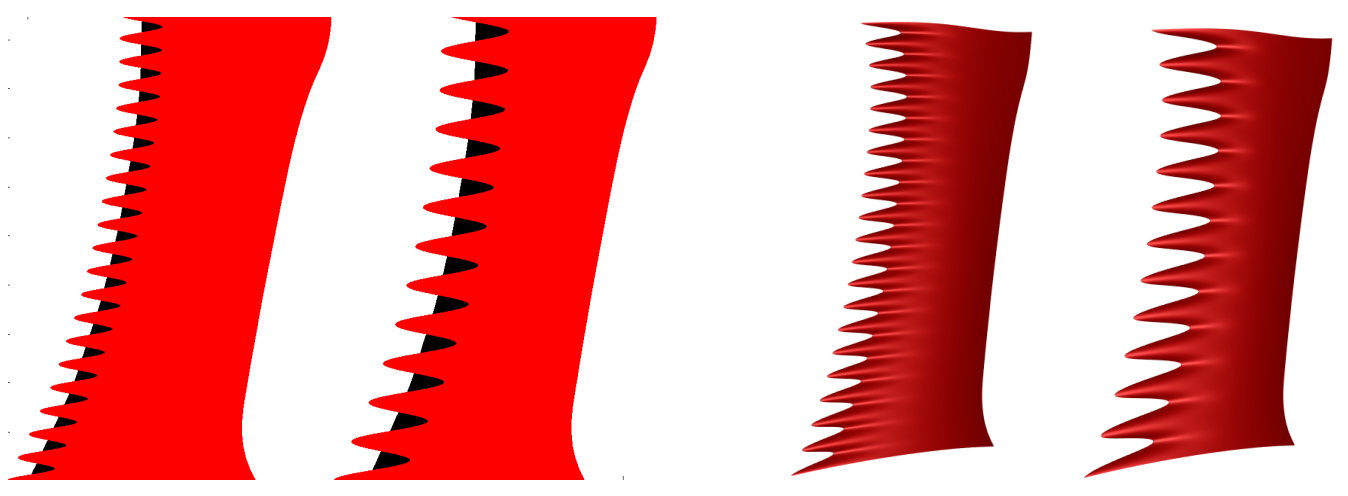

FIG. 7. Initial designs of serrated OGVs (20-waves "PopeUnifom" and 12-waves "GanzUniform"): 2D flat plate (left) and actual 3D (right) geometries.

TABLE I. Strouhal number values (based on $h_{s}$ ) and expected PWL reduction of the "GanzUniform" OGV for the 3 OP.

\begin{tabular}{|c|c|c|c|c|c|}
\hline OP & $U(\mathrm{~m} / \mathrm{s})$ & $f_{\text {peak }}(\mathrm{Hz})$ & $h_{s}(\mathrm{~mm})$ & $f_{\text {peak }} h_{s} / U$ & $\Delta P W L(\mathrm{~dB})$ \\
\hline APP & 120 & 2000 & 16 & 0.27 & 4.3 \\
\hline CUTB & 173.5 & 3500 & 16 & 0.32 & 5 \\
\hline SDL & 193 & 4500 & 16 & 0.37 & 5.7 \\
\hline
\end{tabular}

Nevertheless, results in Table I are probably over-estimated due to the variations of the turbulence characteristics along the OGV span, which highlights a limitation of the radially constant TLS assumption. For this reason, a new design with radial 
variations in the serration parameters has been studied. Local values of $h_{s}$ and $\lambda_{s}$ are set by using a strip approximation of continuous sinusoidal profiles from the radial distribution of TLS in Fig. 6. Furthermore, additional constraints have been set to improve the aerodynamic and structural properties: (i) a local cancellation of the undulations $\left(h_{s}=0\right)$ near the hub, where TI levels are reasonably small and aerodynamic losses can be significant, and (ii) a limited serration amplitude using the criterion $h_{s} / c \leq 0.15$, which is consistent with the threshold set in a from previous work ${ }^{7}$ in collaboration with Safran Aircraft Engines. These additional constraints inspired the final "PopeUniformOpt" and "GanzRadialOpt" designs of an OGV with leading edge serrations (Fig. 8), and have led to a joint patent application between Safran Aircraft Engines and ONERA. These two designs were obtained from the assessment of the aerodynamic performance from various OGVs with LE serrations, as discussed in Section V.
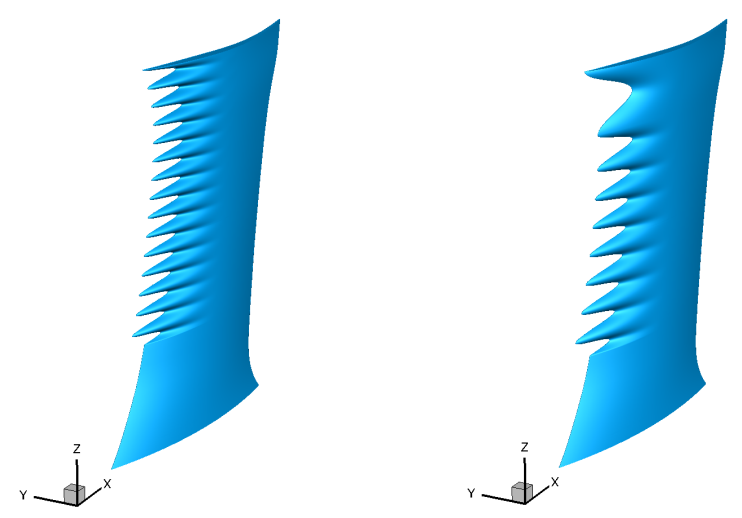

FIG. 8. Final designs for the low-noise OGV using locally distributed leading edge serrations with constant (PopeUniformOpt, left) and spanwise-varying (GanzRadialOpt, right) wavelengths.

\section{CHECKING OF AERODYNAMIC PERFORMANCES AND DOWN SELECTION OF LOW-NOISE OGVS}

The aerodynamic behavior of 5 different designs (see Fig. 9) has been checked by means of RANS mixing plane calculations and compared to the RANS solution for the baseline OGV with straight LE. The aim is to ensure that the impact on the overall aerodynamic efficiency is reduced at ADP.

RANS simulations are performed with the $k-\ell$ Smith model at ADP. The mesh was designed by applying a 3D morphing technique near the undulations using ONERA's in-house modeler (ersatZ) and ensuring at least 40 points per serration wavelength in the spanwise direction. The fan pressure ratio (FPR) and isentropic efficiency are computed upstream and downstream of the fan alone and fan-OGV stage. The FPR-mass flow characteristics of the different configurations are given in Fig. 10 (left). Fan alone performances are not affected by the presence of a serrated OGV. In contrast, the fan-OGV stage performance can vary due to aerodynamic losses through the different OGV concepts. Although the OGVs with LE serrations systematically show a lower FPR than the baseline, a significant reduction of the aerodynamic loss is obtained with 
the modifications applied on "PopeUniformOpt" and "GanzRadialOpt" designs. These two configurations show very similar stage FPR performances that are relatively close to the baseline configuration. For example, the maximum FPR for the OGVs with LE serrations can reach up to 1.42 , whereas it is 1.43 for the baseline. The radial distributions of the OGV loss coefficient are plotted in Fig. 10 (middle) for a particular OP near the maximum efficiency and ADP mass flow rate. Radial locations where the "PopeUniformOpt" and "GanzRadialOpt" configurations mitigate the aerodynamic loss can be clearly identified. This trend is confirmed on isentropic efficiency profiles in Fig. 10 (right), which shows a small impact of the LE serrations for these two designs. A conclusion of this analysis is that the loss reduction of the optimized configurations is obtained from the cancellation of the undulations below 20\% of span (significant improvement in this region compared to "GanzRadial") and the limitation of the serration amplitude (almost the same level of loss as the reference configuration above $80 \%$ of height). RANS calculations performed at APP OP confirmed this behavior with a negligible impact on aerodynamic stability and limited impact on isentropic efficiency compared to ADP case. The maximum penalty was found to be equal to -0.7 pt. at ADP, but it is decreased to -0.58 pt. at APP OP.

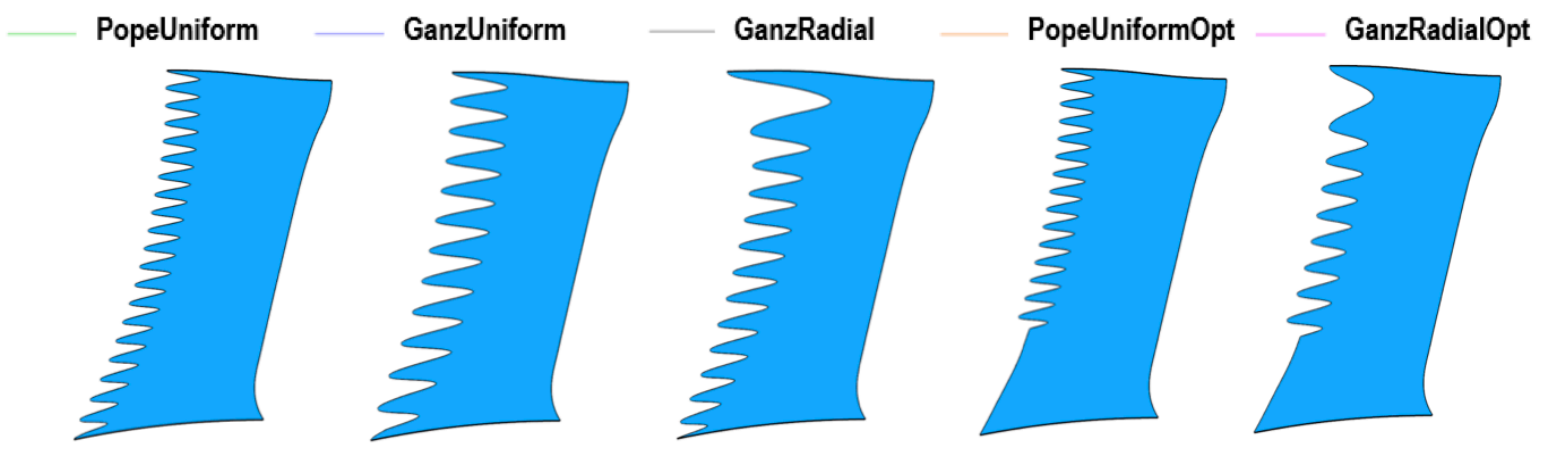

FIG. 9. 5 serrated OGVs selected for the assessment of aerodynamic performances at ADP from RANS simulations.
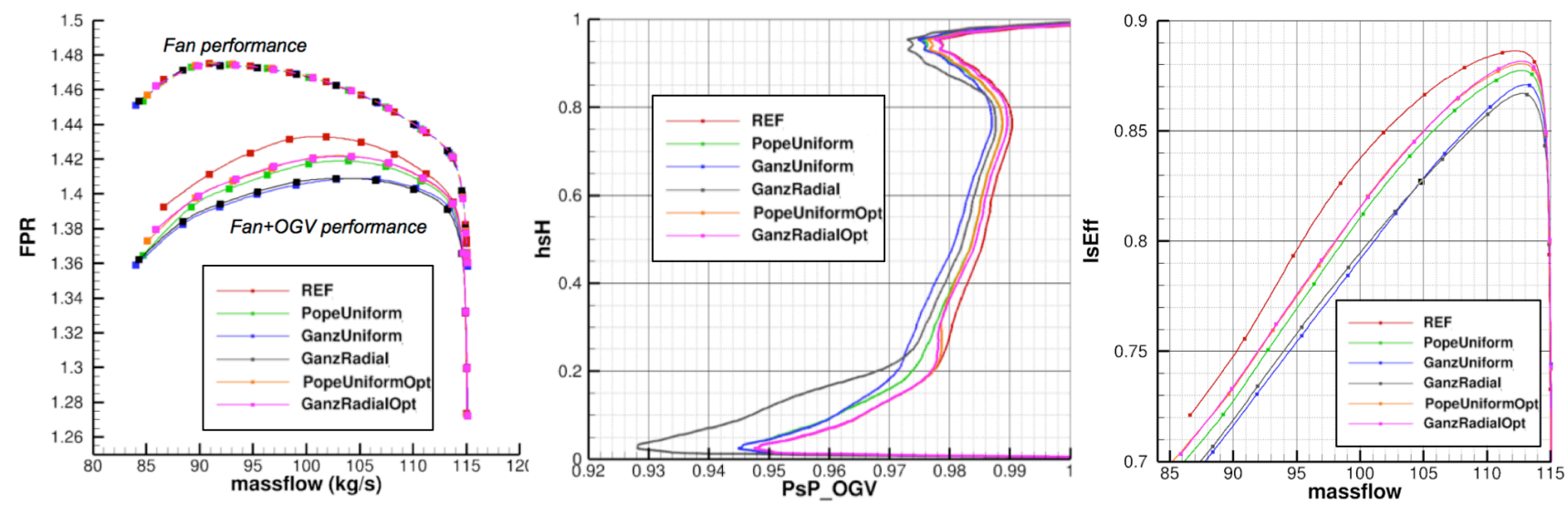

FIG. 10. Comparisons of FPR-massflow characteristics (left), radial distribution of loss coefficient (mid) and isentropic efficiency (right). 


\section{NUMERICAL SIMULATIONS OF BROADBAND NOISE AND ACOUSTIC PERFORMANCES}

This section presents CAA simulations of the baseline and the "GanzRadialOpt" OGVs at approach OP. To this end, 3D numerical simulations are performed using ONERA CAA code $s$ Abrin ${ }^{26}$ with a synthetic turbulence method. This CAA code follows the methodology detailed in Ref. ${ }^{17}$, which has been successfully applied in a previous work [Ref. ${ }^{10}$ ].

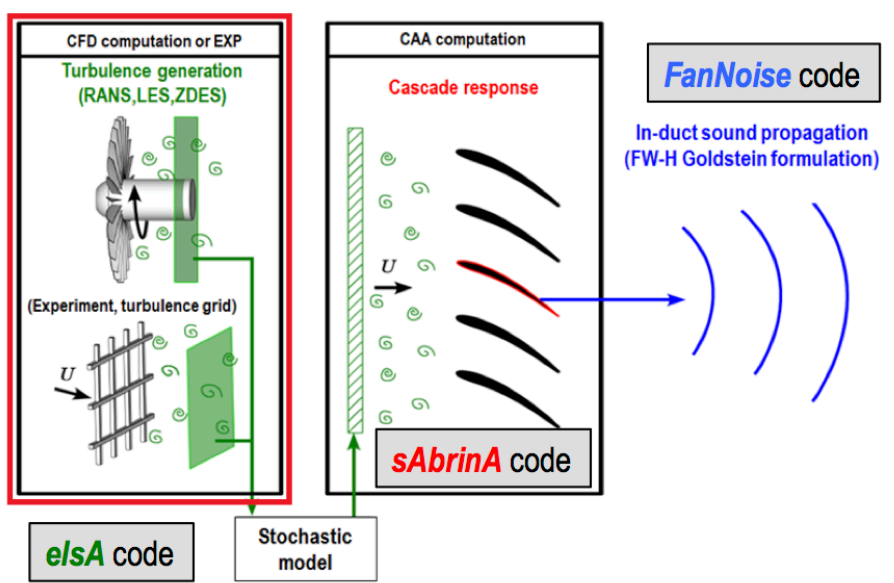

FIG. 11. Schematic of present CAA methodology with the chaining of ONERA codes elsA (RANS), sAbrinA (LEE) and FanNoise (FWH).

\section{A. CAA methodology}

The 3-step hybrid method adopted here to assess the broadband noise $(\mathrm{BBN})$ from the OGVs is presented in Fig. 11. sAbrinA is a CAA code that solves the Euler equations in the time domain with a perturbation form that consists in a splitting of the conservative variables into a mean flow and a disturbance field. The spatial derivatives are computed using a 6th-order finite difference scheme, and a 10th-order explicit filter is applied to remove high-frequency oscillations. The time marching scheme corresponds to a 3rd order Runge-Kutta scheme. The linearized Euler equations (LEE) are adopted here, and a synthetic turbulence model is implemented in order to reproduce the turbulent flow characteristics from the fan wakes. Synthetic turbulence is injected at the inlet of the CAA domain using Tam's boundary condition.

${ }^{27}$ The synthetic turbulence is based on a simple Fourier mode decomposition technique applied to the velocity fluctuations, whose amplitudes are calibrated using a fully isotropic turbulence spectrum model (Liepmann or von-Karman). By analogy to Amiet's theory ${ }^{28}$, only the upwash component of the turbulent flow, $u_{n}$, is modeled, and it is convected by the mean flow as a frozen turbulence. The spatio-temporal harmonic gusts, $u_{n}(x, y, z, t)$, take the form:

$$
u_{n}(x, y, z, t)=2 \int_{0}^{\infty} \int_{-\infty}^{+\infty} \int_{-\infty}^{+\infty} A\left(k_{x}, k_{y}, k_{z}\right) \cos \left(k_{x} x+k_{y} y+k_{z} z-\omega t+\varphi\left(k_{x}, k_{y}, k_{z}\right)\right) d k_{x} d k_{y} d k_{z}
$$


In Eq. (3), $A$ is the mode amplitude, $\omega=k_{x} U_{x}$ is the angular frequency, $\left(k_{x}, k_{y}, k_{z}\right)$ are the hydrodynamic mode wavenumber components, and $\varphi$ is a random phase which can take a value between $[0-2 \pi]$.

The mean square value of $u_{n}$ is related to the 3 -wavenumber isotropic turbulence spectrum, $\phi_{u_{n} u_{n}}$, as follows:

$$
\bar{u}_{n}^{2}=\int_{-\infty}^{\infty} \int_{-\infty}^{+\infty} \int_{-\infty}^{+\infty} \phi_{u_{n} u_{n}}\left(k_{x}, k_{y}, k_{z}\right) d k_{x} d k_{y} d k_{z}
$$

For turbomachinery applications, the initial formulation in Cartesian coordinates has been extended ${ }^{29}$ using cylindrical coordinates, and following the work of Atassi et al. ${ }^{30}$ and Hixon et al. ${ }^{31}$, in order to take into account the swirl and the convection of the gusts along the streamlines (streamwise velocity component, $U_{s}$ ). Introducing the stagger angle $\chi$, assumed to be equal to the absolute flow angle as shown in Fig. 12 , the velocity components in the cylindrical coordinates $(x, r, \theta)$ can be expressed as a function of the upwash velocity fluctuation that is normal to the chord. Denoting $\wedge$ the spatial Fourier transformation, and neglecting the mean radial velocity, as occurs in the OGV stage, the discretized form of the threedimensional synthetic turbulent velocity, associated with the hydrodynamic wavenumber components $\left(k_{x}, k_{r}, k_{\theta}\right)$, can be written as:

$$
\left\{\begin{array}{l}
\hat{u}_{x}=-\frac{k_{\theta}}{k_{\xi}} \hat{u}_{n} \\
\hat{u}_{\theta}=\frac{\hat{u}_{n}}{\cos (\chi(r))}\left(1-\frac{k_{\theta} U_{\theta}}{\omega}\right) \\
u_{n}(x, r, \theta, t)=2 \sum_{n=1}^{N} \sum_{m=-M}^{M} \sum_{l=-L}^{L} A_{n m l} \cos \left(k_{x, n} x+k_{r, m}\left(r-r_{h}\right)+m_{g, l} \theta-\omega_{n} t+\varphi_{n m l}\right)
\end{array}\right.
$$

In Eq. (5), $r_{h}$ is the hub radius, $U_{\theta}$ is the circumferential velocity component, and $A_{n m l}$ is the gust amplitude which is related to the turbulence spectrum as:

$$
A_{n m l}=\sqrt{\phi_{u_{n} u_{n}}\left(k_{\xi, n}, k_{r, m}, k_{\theta, l}\right) \Delta k_{\xi} \Delta k_{r} \Delta k_{\theta}} \text {, with } k_{\xi}=\frac{\omega}{U_{s}}=k_{x} \cos (\chi), k_{\theta}=\frac{m_{g}}{r}, \Delta k_{\xi}=\Delta k_{x} \cos (\chi), \Delta k_{\theta}=\frac{\Delta m_{g}}{r}
$$



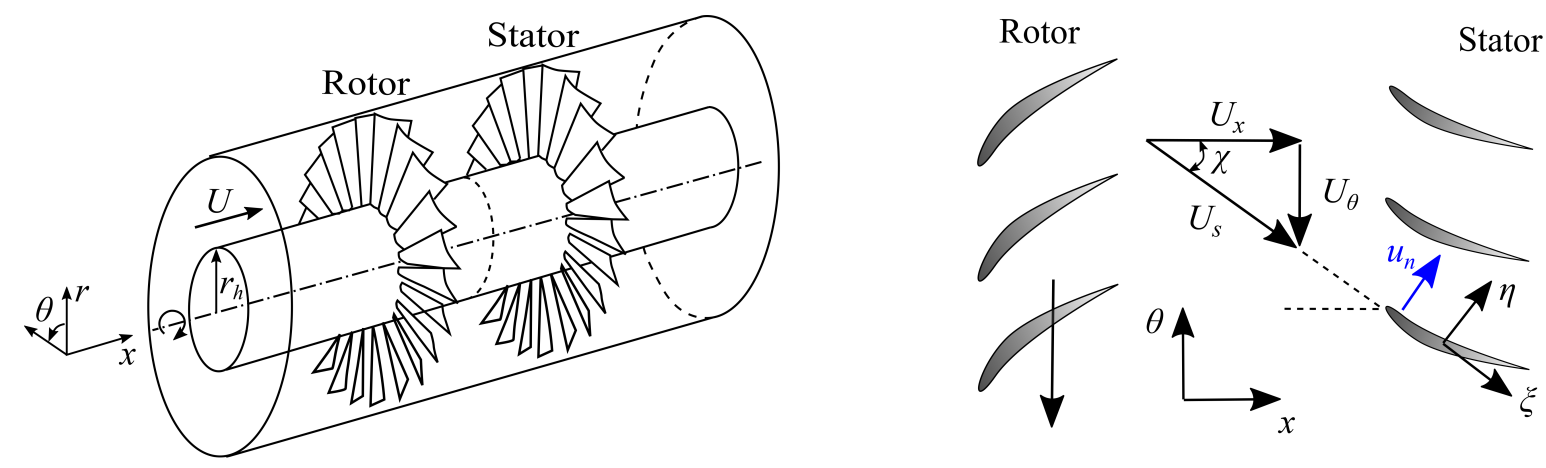

FIG. 12. Representation of an annular cascade (left) and velocity diagram of the rotor-stator interstage (right).

As discussed in Ref. ${ }^{17}$, fully 3D simulations are still challenging and very expensive in terms of CPU cost, as it requires a $360^{\circ}$ domain (or at least several vane-channels satisfying the condition $\Delta m_{g}=2 \pi / \Delta \Psi$, where $\Delta \Psi$ is the periodicity sector angle). In practice, the CAA domain can be restricted to a single vane-channel (with periodic boundary condition in the circumferential direction) by considering a 2-wavenumber turbulence spectrum, $\phi_{u_{n} u_{n}}\left(k_{\xi}, k_{r}\right)$. The latter can be obtained from an integration over the angular wavenumbers, so that the injected synthetic turbulence field is axisymmetric. Hence, the two-dimensional synthetic velocity field can be written as:

$$
\left\{\begin{array}{l}
\hat{u}_{x}=0 \\
\hat{u}_{\theta}=\frac{\hat{u}_{n}}{\cos (\chi(r))} \\
u_{n}(x, r, t)=2 \sum_{n=1}^{N} \sum_{m=-M}^{M} A_{n m} \cos \left(k_{x, n} x+k_{r, m}\left(r-r_{h}\right)-\omega_{n} t+\varphi_{n m}\right) \\
A_{n m}=\sqrt{\phi_{u_{n} u_{n}}\left(k_{\xi, n}, k_{r, m}\right) \Delta k_{\xi} \Delta k_{r}}
\end{array}\right.
$$

This simplified approach is preferred here, and the CAA code with the above-mentioned synthetic turbulence is used to assess the unsteady wall pressure over the stator vane. This information is used as in input to a Ffowcs-Williams and Hawkings (FWH) solver that computes the sound field radiated in the bypass duct. In-duct propagation is modeled by introducing a Green's function that is valid for a uniform mean flow and a semi-infinite annular duct, as proposed by Goldstein. ${ }^{32}$ This technique leads to an usual expansion over Fourier-Bessel modes solved by using the propagation code FanNoise $^{33}$, in which the fan shielding effects are fully neglected.

\section{B. RANS-derived information and CAA set-up}


Two different RANS simulations are required to feed the CAA method: (1) one fan-OGV simulation using a mixing plane (see Fig. 3, left) is necessary to provide the mean flow for the CAA simulation; (2) one fan alone simulation (Fig. 3, right) to extract the turbulence flow information (TI and TLS) near the LE plane of the OGV. The mean flow from the RANS mixing-plane calculation is interpolated onto the CAA grid and is used as a background flow for the CAA simulations using sAbrinA's code. As an illustration, Fig. 13 shows the axial velocity field in the fan-OGV stage. The turbulence statistics from the fan alone RANS solution are used to calibrate the turbulent velocity spectrum, $\phi_{u_{n} u_{n}}$, given by the Liepmann model:

$$
\phi_{u_{n} u_{n}}\left(k_{\xi}, k_{r}\right)=\frac{3 \bar{u}_{n}^{2} \Lambda^{2}}{4 \pi} \frac{\left(k_{\xi}^{2}+k_{r}^{2}\right) \Lambda^{2}}{\left[1+\left(k_{\xi}^{2}+k_{r}^{2}\right) \Lambda^{2}\right]^{5 / 2}}
$$

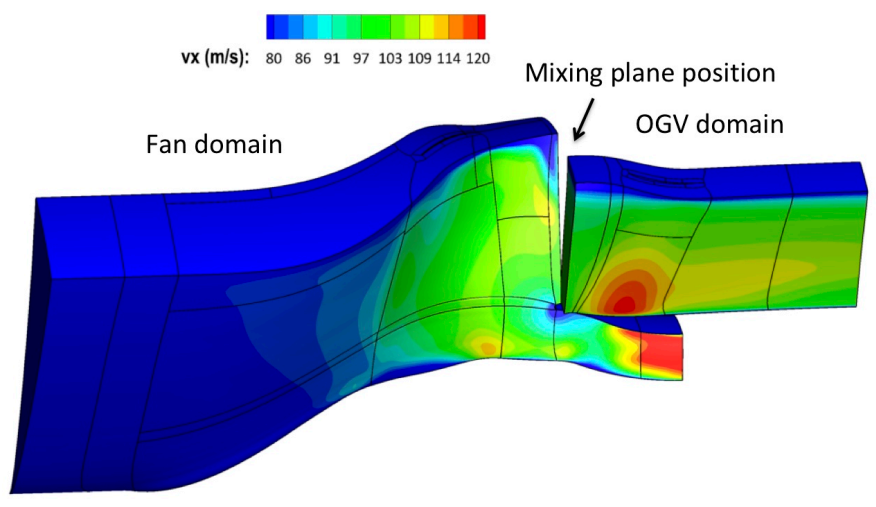

FIG. 13. Axial velocity contours $(\mathrm{m} / \mathrm{s})$ in the fan-OGV stage provided by a RANS simulation at APP OP using $k-\ell$ turbulence model.

Three-dimensional views of the CAA domain and blocking topology are shown in Fig. 14. In Fig. 14 on the left, the domain has been duplicated over 11 channels, as required for simulations based on a 3-wavenumber turbulence spectrum. ${ }^{17}$ The CAA domain is shown in Fig. 14 on the right, and it is colored by axial mesh spacing deformation for the serrated OGV. Sensitivity of the method to the mesh density has been studied in previous work ${ }^{7,17}$, by considering coarse, medium and fine grid resolutions on the NASA SDT benchmark case $\left(\right.$ Ref. $\left.^{7}\right)$, and by adjusting the refinement in axial and radial directions on the baseline ACAT1 model (Ref. ${ }^{17}$ ). At least 15 points per wavelength are used to ensure an accurate convection of the gusts along the streamlines. The mesh is highly refined in the leading edge region to well capture the leading edge noise sources, and in the trailing edge region too, in order to safisfy the Kutta condition. A special care has been paid to the CAA set-up in order to keep the same grid density for the baseline and serrated OGV cases while limiting the computational cost. A highly refined mesh is necessary to accurately discretize the serrations ( 40 points per sinusoidal pattern are used, wheah leads to 800 points along the span), so that a single vane passage in the CAA domain has about 37 million grid points. Several views of CAA grids are presented in Fig. 15, showing the mesh refinement in the vicinity of the vane wall and the accurate spatial 
discretization of the serration patterns. In addition to the Tam's outlet non-reflecting boundary condition ${ }^{27}$, a stretched grid zone is added at the outlet boundary to damp the outgoing disturbances.

Simulations were run with a frequency resolution of $\Delta f=250 \mathrm{~Hz}$ (time period of $1 / 250 \mathrm{~s}$ ) and $f_{\max }=10 \mathrm{kHz}$, requiring about 80 hours of CPU time (160, 000 iterations per period over 657 processors) to get a converged solution.
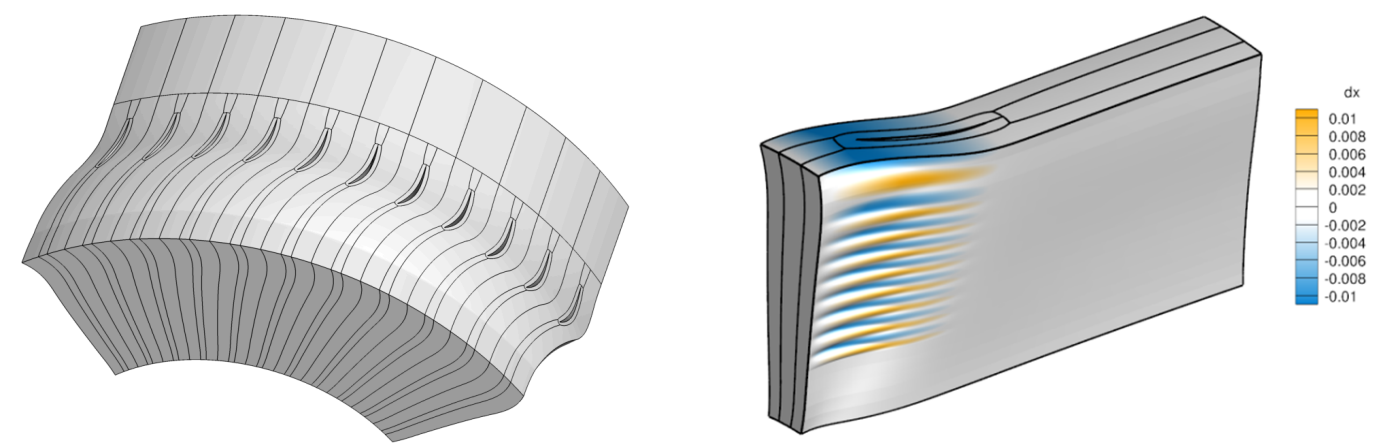

FIG. 14. CAA blocking topology for a 11-channels domain (left) and a 1-channel domain with axial grid spacing deformation for a serrated OGV (right)
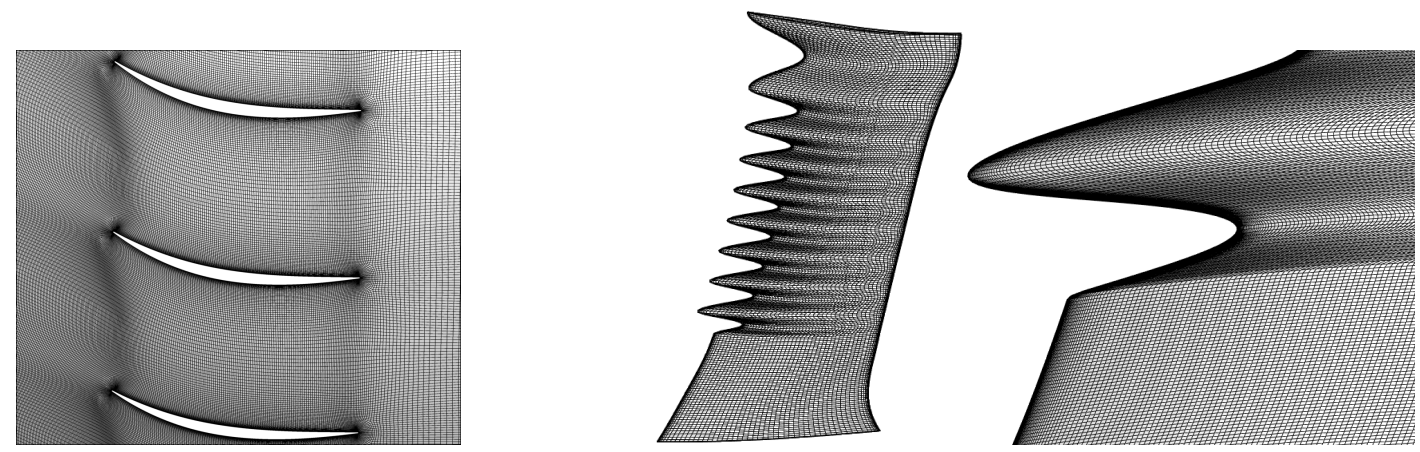

FIG. 15. Circumferential mesh of the unwrapped cascade (left) and skin mesh of a serrated vane (1 over 4 pts.) with a close-up of the first serration near the hub (right)

Statistical errors in noise spectra calculations are minimized by applying a frequency-band averaging technique. It should be noted that the number of time iterations that is necessary to obtain converged results depends on the CFL $<1$ condition, which ensures the stability of the simulation. Thus, the number of time iterations increases linearly with the number of desired averages, and have a significant impact on the computational expense. Here, the number of averages without overlapping is restricted to 3 . Based on the results from a previous simulation ${ }^{17}$ on the baseline case using a 4 -channels domain (to better capture possible cascade effects) with a moderate grid density in the radial direction (around 100 points) and a $100 \mathrm{~Hz}$ frequency spacing resolution, it has been preferred to limit the frequency resolution to $250 \mathrm{~Hz}$ for the present CAA simulation. Moreover, due to strong mean flow gradients generated at the trailing edge (TE) in the hub region ${ }^{10}$ which are not convenient for CAA simulations using the slip wall conditions and the Euler equations, the background flow was locally adjusted to obtain reliable CAA results that are presented in Section C. 


\section{Simulation results}

CAA simulations in Ref. ${ }^{10}$ have encountered several issues due to strong mean flow gradients, which could generate spurious noise sources and diverging local instabilities, particularly in the presence of LE serrations. Improved CAA solutions have been recently obtained through an iteration process that consists in a local correction of the RANS mean flow to remove both the boundary layers at the wall and flow inhomogeneities at the outflow plane, in order to satisfy the slip wall and Tam's outlet boundary conditions in the CAA simulations, respectively. An example of the mean flow adjustments is given in Fig. 16, showing a separation buble removal near the OGV TE (Fig. 16, left) and a correction for the velocity field asymmetry at the outlet plane (exhaust boundary) of the CAA domain (Fig. 16, right). The benefit of these modifications is clearly highlighted in Fig. 17, which shows the root-mean-square (RMS) wall pressure field on the serrated vane suction side obtained from CAA with initial (Fig. 17, left) and adapted (Fig. 17, right) RANS mean flows. A major improvement can be seen near the TE and hub regions, where the spurious noise sources are significantly reduced from the CAA simulation. This process was used to obtain the most reliable numerical predictions presented and discussed here after.
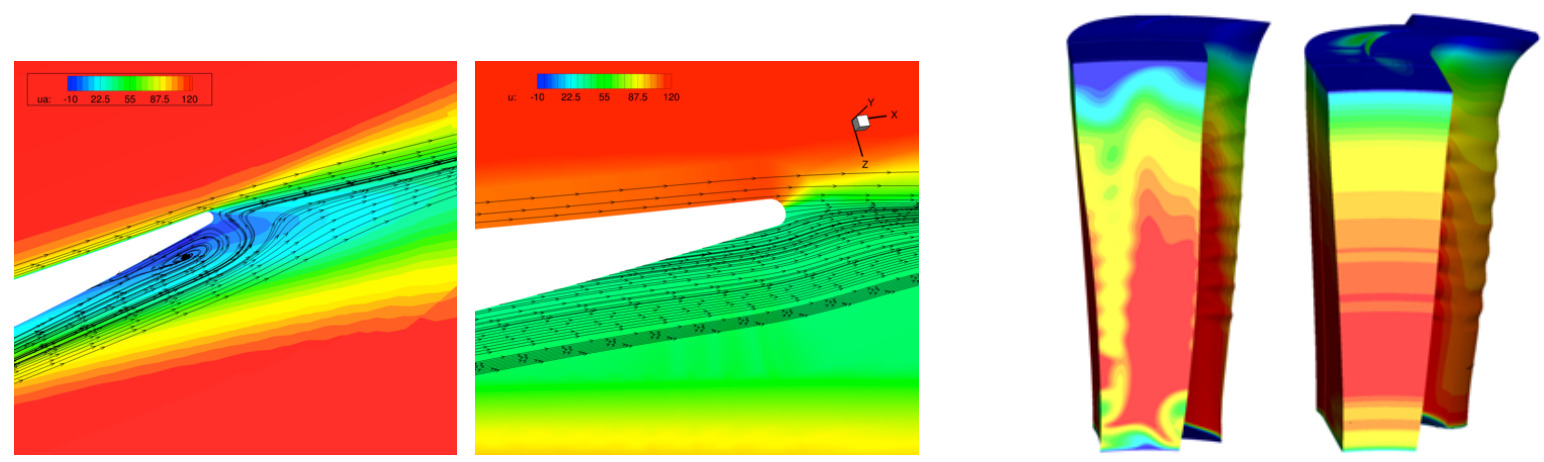

FIG. 16. Mean flow corrections for CAA simulations: recirculation buble with streamlines over a cut at mid-span and a zoom around the $\mathrm{TE}$ (left) and inhomogeneous axial velocity at the outflow plane (right).
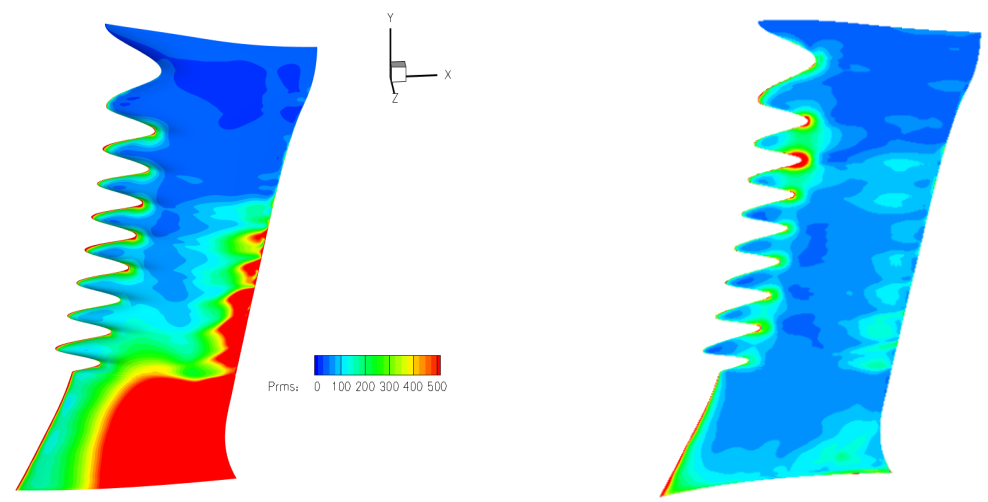

FIG. 17. RMS pressure contours (Pa) on the "GanzRadialOpt" vane suction side: initial CAA solution (left) and last solution using an adapted mean flow (right).

Fig. 18 shows instantaneous contours of the tangential velocity disturbances using synthetic turbulence for the "GanzRadialOpt" OGV simulation. CAA results were obtained after two overall periods $T_{s i m}\left(T_{s i m}=1 / \Delta f\right)$, which is enough to 
obtain well converged noise and turbulence statistics. It should be noted that only the second period is stored for the acoustic post-processing.

Comparisons between baseline and serrated OGV simulations are shown in terms of RMS wall pressure in Fig. 19. Noise sources are mainly located in the LE region for both cases, as expected when LE noise is dominant. The noise sources at the LE are uniformly distributed along the span for the baseline case, whereas they are concentrated at the roots of the serrations, as observed on isolated airfoils ${ }^{2,4-6}$. The straight LE region of the serrated vane near the hub displays similar levels to the baseline ones, which is consistent. The significant attenuation of the RMS pressure along the hills of the serrated OGV should certainly provide a non-negligible noise reduction that can be evaluated with the in-duct FWH code. Nevertheless, some remaining spots at the TE are still visible on the serrated suction side, which are believed to be spurious sources (see previous discussion related to Fig. 17). Consequently, FWH calculations have been performed by limiting the integration surface of the vane to exclude the unphysical sources present in the TE region and in the vicinity of the hub. Fig. 20 shows the integration surface for the FWH calculations, where the dotted regions have been excluded for accuracy purposes. In practice, the neglected zones are roughly set equal to $10 \%$ of the span from the foot and $30 \%$ of the chord from the TE. The same integration surface is considered for both the baseline and serrated cases.
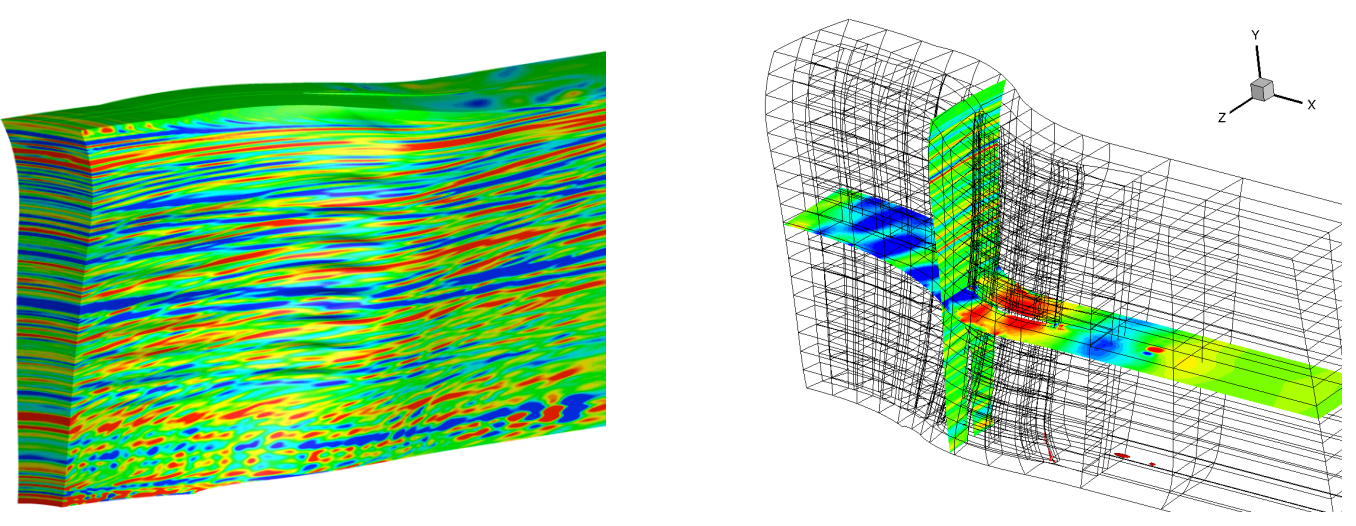

FIG. 18. 3D (left) and 2D (right) instantaneous contours of tangential velocity disturbances.
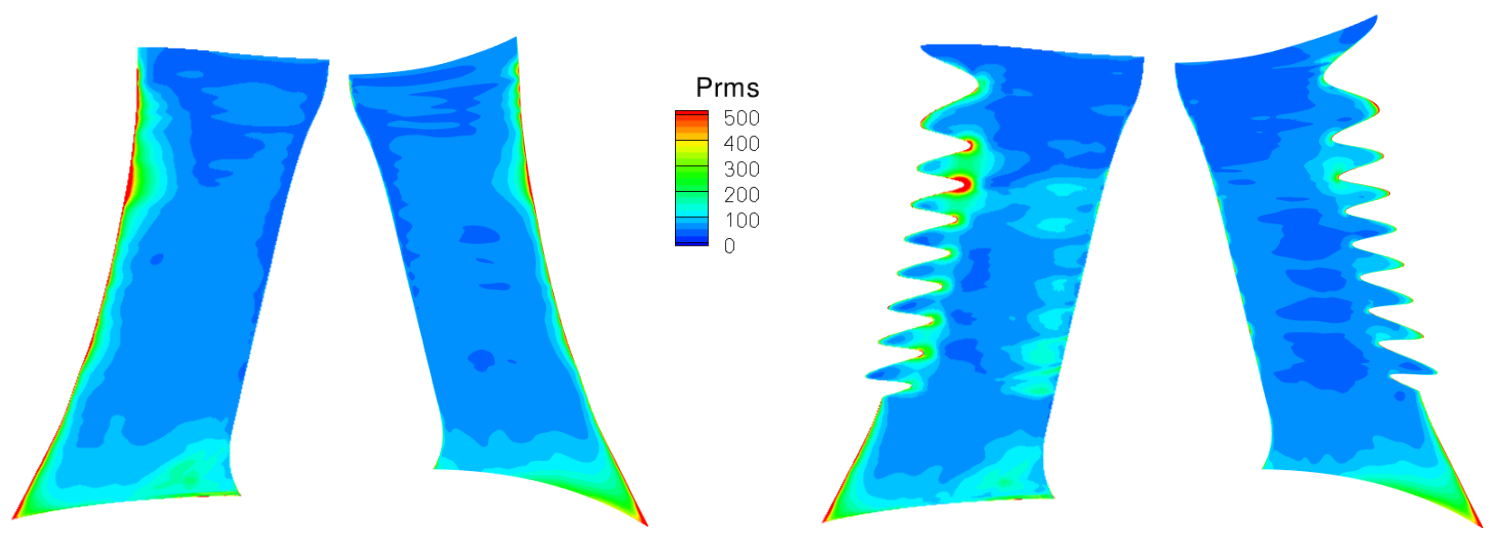

FIG. 19. RMS pressure contours on the suction and pressure sides obtained for the baseline (left) and serrated (right) LE vanes. 

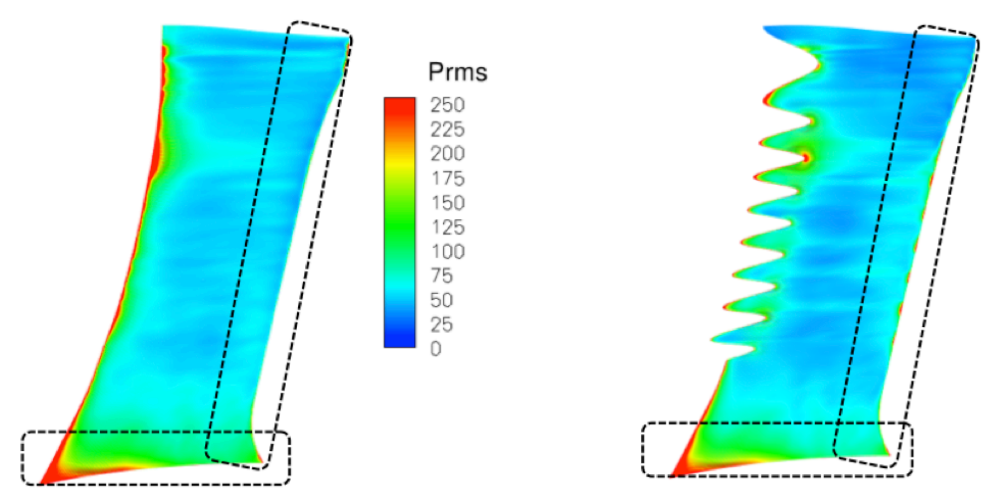

FIG. 20. RMS pressure contours and FWH integration surface for the baseline (left) and serrated (right) LE cases. The dotted regions are neglected in the FWH calculations.

PWL spectra in the bypass duct (downstream of the OGV) provided by FanNoise are shown in Fig. 21 (left), using full and limited FWH integration surfaces. The effect of the regions with spurious noise sources is quite significant and has an impact on the spectrum shape, particularly at high frequencies, where a slope change can be observed from 3-4 kHz. The PWL reduction (delta PWL) obtained with the serrations is emphasized when using the limited FWH integration surface. The benefit of this approach is confirmed in Fig. 21 on the right, which shows a comparison of the baseline CAA prediction (using limited integration surface) with the previous CAA solution ${ }^{17}$ and with experimental measurements. A good agreement can be observed between the simulation and experimental results in terms of spectral shapes, where close levels between numerical solutions are found. One should note that the baseline result from Ref. ${ }^{17}$ was obtained using a 4-channels CAA domain to account for the cascade effect which is partly neglected when applying periodicity conditions on a single vane channel. The cascade effects are known to decrease the sound levels at low and medium frequencies, which can balance the level attenuation due to the surface truncation. Nevertheless, the relative PWL deviation between baseline and serrated OGV cases is assumed to be reliable.

Variations in delta PWL are presented in Fig. 22 by adopting an additional FWH integration surface with a slightly enlarged truncation: $12.5 \%$ of the span (instead of $10 \%$ ) and $33 \%$ of the chord (instead of $30 \%$ ). Although the absolute power levels are reduced when extending the truncated region on both the baseline and serrated LE vanes (Fig. 22, left), the relative PWL deviation appears to be more pronounced as shown on the delta PWL spectra plotted in Fig. 22, on the right. The acoustic performances provided by the numerical predictions with the second truncation set-up are believed to be more relevant, as the main noise sources and noise reduction mechanism are located in the LE region. Hence, an OAPWL reduction of at least $2 \mathrm{~dB}(2.3 \mathrm{~dB}$ obtained from the thick line spectrum integration) can be expected from our numerical predictions for the "GanzRadialOpt" low-noise OGV at the approach OP. 

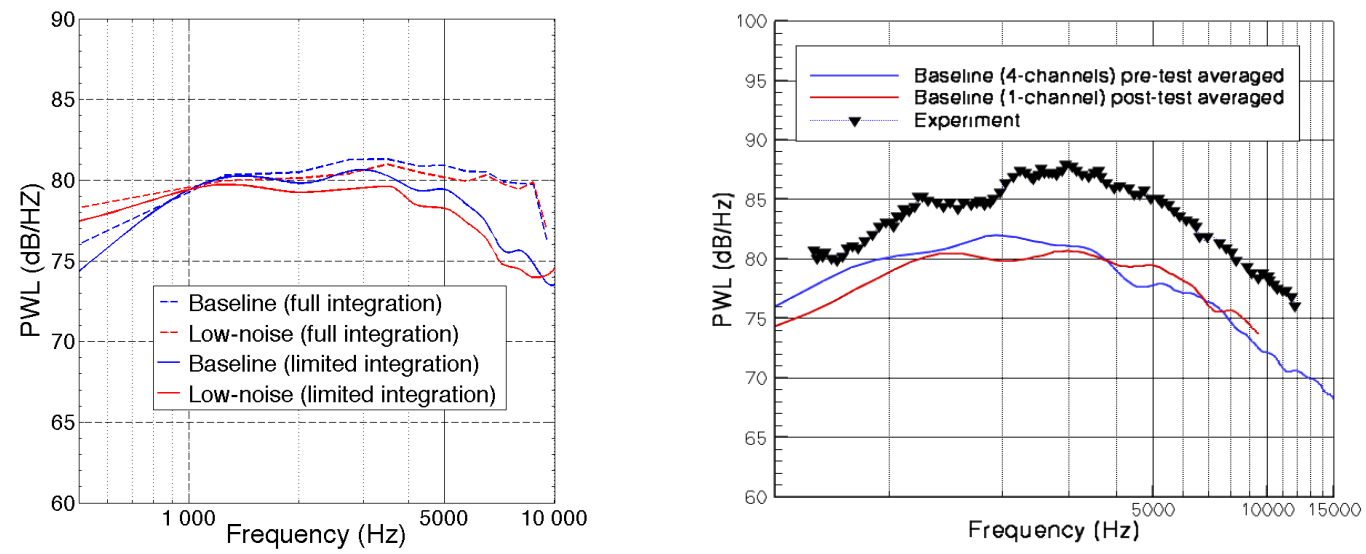

FIG. 21. PWL spectra (bypass duct) provided by the CAA+FWH methodology using both full and limited integration surface (left) and comparison with a previous numerical solution (from Ref. ${ }^{17}$ ) and experimental measurements (right).
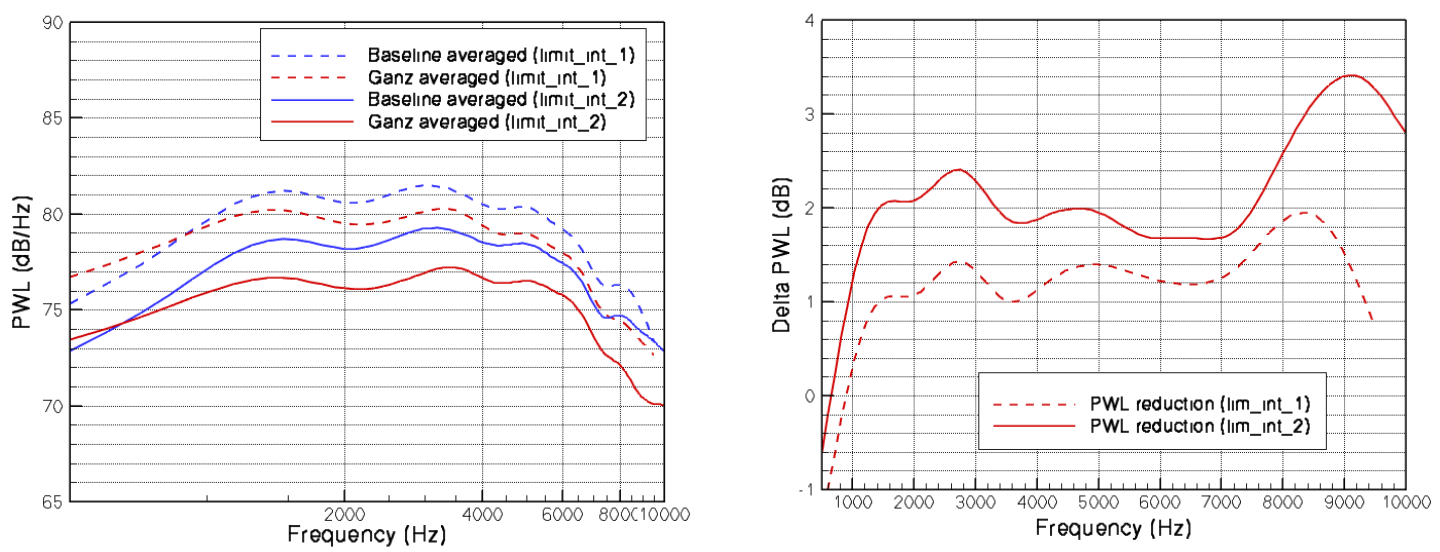

FIG. 22. PWL spectra (left) and delta PWL spectra (right) obtained from CAA+FWH solutions for baseline and serrated OGVs with two selected (limited) integration surfaces.

\section{WIENER-HOPF PREDICTIONS OF NOISE REDUCTION}

This section presents an investigation based on an analytical model that has been recently proposed. This study is helpful to confirm some of the numerical results, and it is motivated by the development of an efficient analytical model ${ }^{8}$ to predict broadband noise from a serrated LE airfoil.

\section{A. Brief overview of the formulation}

This part presents the analytical solution proposed by Ayton and $\mathrm{Kim}^{8}$ and Lyu et al. ${ }^{34}$ for the calculation of the far-field noise generated by a gust-plate interaction including LE serrations (as shown in Fig. 23). The main specificity of this formulation is to take into account any serration shape with a periodical pattern. This is useful to estimate the broadband noise reduction associated with the sinusoidal LE serrations previously discussed. The analytical model makes the following assumptions:

- Uniform convective velocity parallel to the chord; 
- Semi-infinite flat plate (in the chordwise and spanwise directions);

- Periodic LE undulations (in this section, only sinusoidal wavy LE plates are considered);

- Acoustic perturbation potential satisfying the Helmholtz convective equation;

- 2D planar wave-number turbulence spectrum.

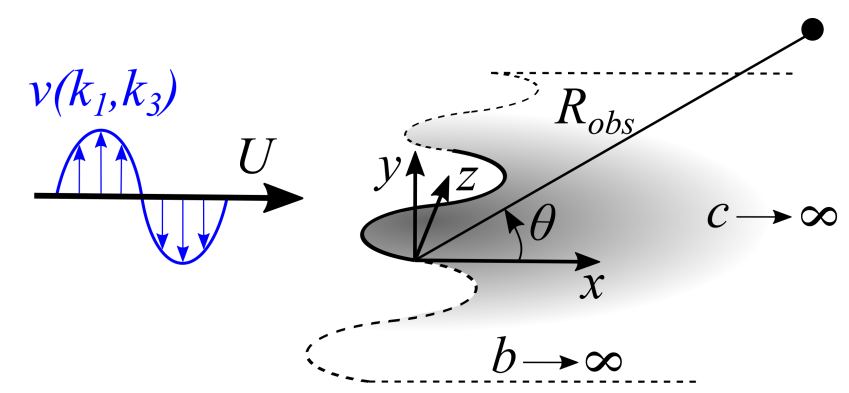

FIG. 23. Schematic illustration of the LE serration and the Cartesian reference frame.

The governing equation to be solved assumes that the pressure perturbation can be derived from a scattered velocity potential, i.e. $p=-\rho_{0} \frac{D \phi}{D t}$, where $\rho_{0}$ is the mean flow density. The problem is sought for harmonic solutions of incoming harmonic gusts normal to the plate and assuming a frozen turbulence, which leads to the convective Helmholtz equation:

$$
\left(1-M^{2}\right) \frac{\partial^{2} \phi}{\partial x^{2}}+\frac{\partial^{2} \phi}{\partial y^{2}}+\frac{\partial^{2} \phi}{\partial z^{2}}+2 i k M \frac{\partial \phi}{\partial x}+k^{2} \phi=0
$$

A detailed derivation of the analytical model using dimensionless variables from Eq. (8) is given in Ref. ${ }^{8}$. The main steps involve the use of convenient variable changes that are adopted to describe the serration over one wavelength with periodic boundary conditions. Then, a Fourier transform is applied on the main unknown function $\left(h\right.$ function in Ref. $\left.{ }^{8}\right)$ and the solution to the governing equations is obtained using the separation variable technique. Finally, the system of equations obtained is solved by using the Wiener-Hopf (WH) technique, and the acoustic field is deduced from an inverse Fourier transform and the infinite limit approximation of the observer distance to get an asymptotic expression for $h$. A more compact form is proposed by Lyu et al. ${ }^{34}$, which relates the radiated sound pressure (in the far-field) as a response to a $2 \mathrm{D}$ gust $v\left(k_{1}, k_{3}\right)$ associated to streamwise, $k_{1}$, and spanwise, $k_{3}$, wavenumbers. Considering an incoming gust $v_{i}$ of the form, $v_{i}=v_{0} e^{i\left(-\omega t+k_{1} x\right)}$, where $v_{0}$ is the gust amplitude, the dimensionless $(\sim)$ far-field sound pressure can be written in a compact form as:

$$
\tilde{p}\left(\tilde{r}, \tilde{\theta}, \tilde{z}, \tilde{k}_{1}, \tilde{k}_{3}\right)=L\left(\tilde{r}, \tilde{\theta}, \tilde{z}, \tilde{k}_{1}, \tilde{k}_{3}\right) \tilde{v}_{0}\left(\tilde{k}_{1}, \tilde{k}_{3}\right)
$$


In Eq. (9), $(r, \theta)$ are the 2D polar coordinates of the observer from a compact source taken at $z$ (for most practical applications $z$ corresponds to the middle of the span and can be set equal to zero), and $L$ is the aeroacoustic response function. The reader is referred to Appendix A for further details on the main steps of the analytical model.

The broadband noise spectrum is obtained from Eq. (9) by integrating over the spanwise wave numbers $\left(k_{3}\right)$, and introducing a 2-wavenumber turbulence spectrum (such as those from von Karman or Liepmann models), $\phi_{22}$, that is related to the upwash velocity disturbance. The dimensional Power Spectral Density (PSD) of the sound pressure in the wavenumber space takes the form:

$$
\left|p\left(k_{1}, r, \theta\right)\right|^{2}=\rho_{0}^{2} U^{2} \int_{-\infty}^{+\infty}\left|\tilde{p}\left(\tilde{k}_{1}, \tilde{k}_{3}, \tilde{r}, \tilde{\theta}\right)\right|^{2} \phi_{22}\left(\tilde{k}_{1}, \tilde{k}_{3}\right) d \tilde{k}_{3}
$$

The PSD in the frequency space, $S(f)$ (in $\mathrm{Pa}^{2} / \mathrm{Hz}$ ), is given by:

$$
S(f, r, \theta)=\frac{2 \pi}{U}\left|p\left(k_{1}, r, \theta\right)\right|^{2}
$$

One should note that the original formulation is written for a semi-infinite span, assuming two-dimensional far-field noise propagation and using a 2D Green's function which varies as a function of $1 / \sqrt{R_{o b s}}$, where $R_{o b s}$ is the distance between the compact source and the observer. Some corrections (that are not explicitly stated in Refs. ${ }^{8,34}$ ) have to be introduced to account for finite span airfoils and 3D far-field radiation. These are necessary to validate the present formulation using the FLOCON database (Section VII.B) and the low-noise OGV design that has been proposed in this paper (Section VII.C). From rather simple geometrical acoustic rules and using Taylor's expansion (see Appendix B), it can be shown that such 2D to $3 \mathrm{D}$ corrections lead to a correction factor of $b / R_{o b s}$, where $b$ corresponds to the airfoil span. This factor $b / R_{o b s}$ seems consistent with the result found by Gea-Aguilera et al. ${ }^{34}$, when applying $2 \mathrm{D}$ to $3 \mathrm{D}$ corrections for Amiet's theory. Thus, the $3 \mathrm{D}$ far-field sound pressure radiated from a centered point-source of the finite span airfoil, $p_{3 D}$, that satisfies the assumption $b<<R_{\text {obs }}$ can be related to the $2 \mathrm{D}$ asymptotic solution ${ }^{34}, p_{2 D}$, as:

$$
\left|p_{3 D}\left(b, R_{o b s}\right)\right|_{\frac{b}{R_{o b s}} \rightarrow 0}^{2} \approx \frac{b}{R_{o b s}}\left|p_{2 D}\left(R_{o b s}\right)\right|^{2}
$$

It should be noted that $p_{3 D} \rightarrow p_{2 D}$ when applying the condition $b \rightarrow \infty$ (see Appendix B).

This extended finite-span formulation is validated in Section VII.B from:

(1) A comparison of broadband noise predictions (from both absolute SPL and PWL spectra) on straight LE airfoils using the present WH approach (with $h_{s}=0$ ) and Amiet's solution; 
(2) A comparison of broadband noise predictions from serrated LE airfoils using CAA and experimental results. ${ }^{2,4}$

\section{B. Validation on FLOCON case}

The extended WH formulation is first applied to a well-documented test-case on the NACA 651210 airfoil ${ }^{2,4}$. Baseline and serrated LE configurations are considered here, and the main parameters are summarized in Table II.

TABLE II. Parameters from FLOCON test-case for turbulence-airfoil noise predictions.

\begin{tabular}{|c|c|c|c|c|c|c|c|}
\hline Chord $(\mathrm{m})$ & $b(\mathrm{~m})$ & TI $(\%)$ & TLS $(\mathrm{m})$ & $h_{s}(\mathrm{~m})$ & $\lambda_{s}(\mathrm{~m})$ & Mach & $R_{\text {obs }}(\mathrm{m})$ \\
\hline 0.15 & 0.45 & 2.5 & 0.006 & $\begin{array}{c}0 \text { (baseline }) \\
0.005\end{array}$ & 0.01 & 0.18 & 1.2 \\
\hline
\end{tabular}

WH predictions of SPL at $\theta=90^{\circ}$ and PWL spectra are compared to Amiet's solution in Fig. 24. Sound power is computed by assuming cylindrical radiation, as proposed in Refs., ${ }^{2,4}$. Overall, a good agreement can be observed although some oscillations are visible in the Amiet-based SPL spectrum. This can be attributed to compactness effects along the chord, which cannot be accounted for in the present WH solution due to the semi-infinite chord assumption.

The WH formulation is applied to the LE serration case, and predictions are compared to available results from CAA and measurements ${ }^{2,4}$. The SPL spectra at $\theta=90^{\circ}$ are presented in Fig. 25 for both baseline and serrated LE cases. A quite good agreement is found although the WH solution tends to under-predict the noise levels at low frequencies. The acoustic performances of the LE serrations is confirmed by the WH approach analytical method in Fig. 26, which shows both delta SPL and delta PWL spectra to highlight the noise reduction. For the present case, the WH analytical model is able to reproduce the trends obtained from CAA and experimental results. The limited acoustic benefit at high frequencies (beyond 3-4 kHz) observed on measurements can be attributed to the TE noise. In Fig. 26, the self-noise contribution has been evaluated analytically following Howe's work ${ }^{36}$, and added to the WH solution. This improves the comparison with experimental results at high frequencies.
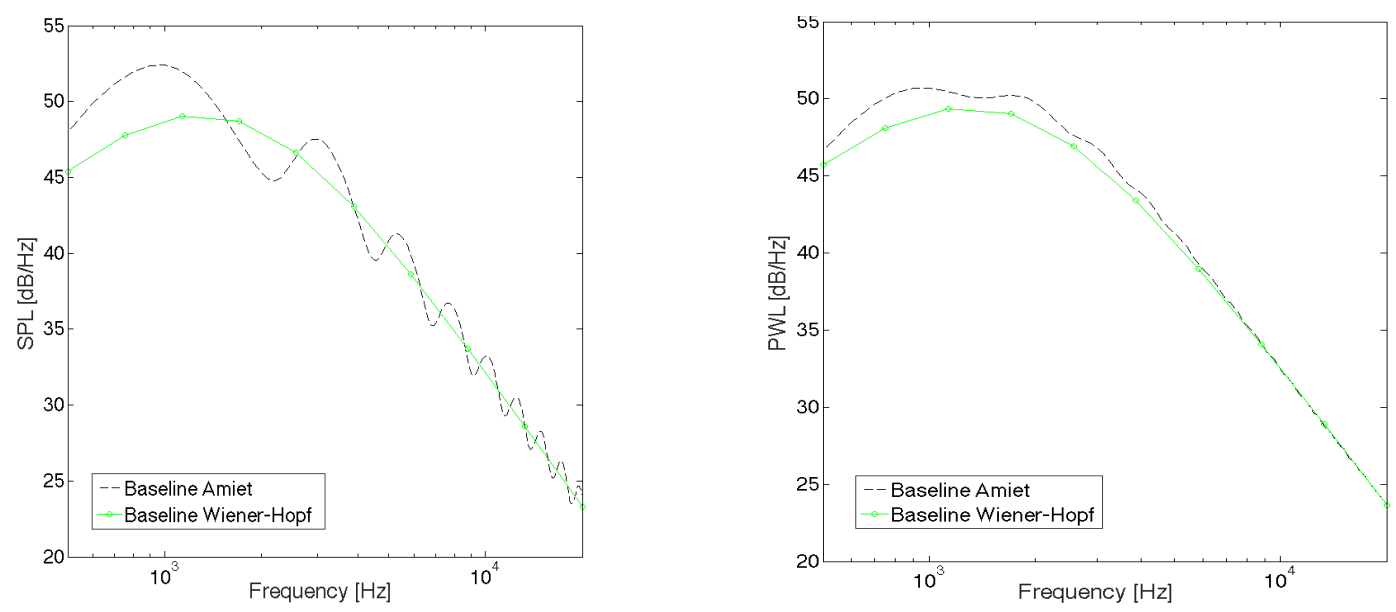

FIG. 24. SPL spectra at $\theta=90^{\circ}$ (left) and PWL spectra (right) from Amiet and WH solutions for the baseline LE flat plate. 


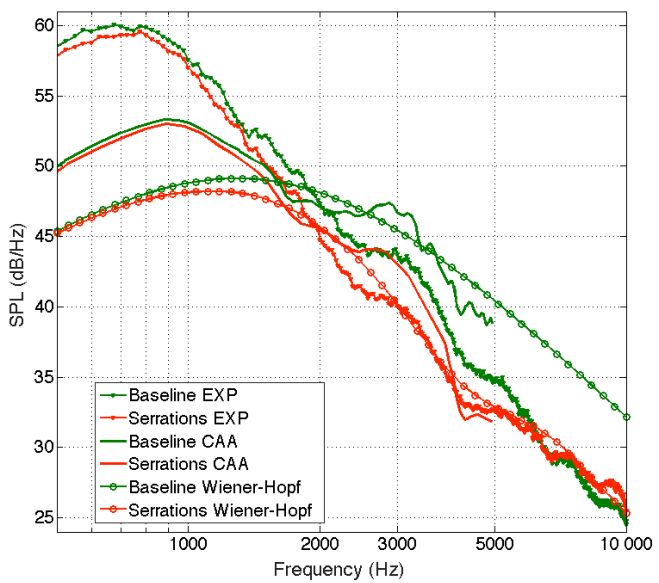

FIG. 25. SPL spectra at $\theta=90^{\circ}$ for baseline and serrated LE cases from CAA solutions ${ }^{2}$, WH analytical model ${ }^{8}$, and experiment ${ }^{4}$.
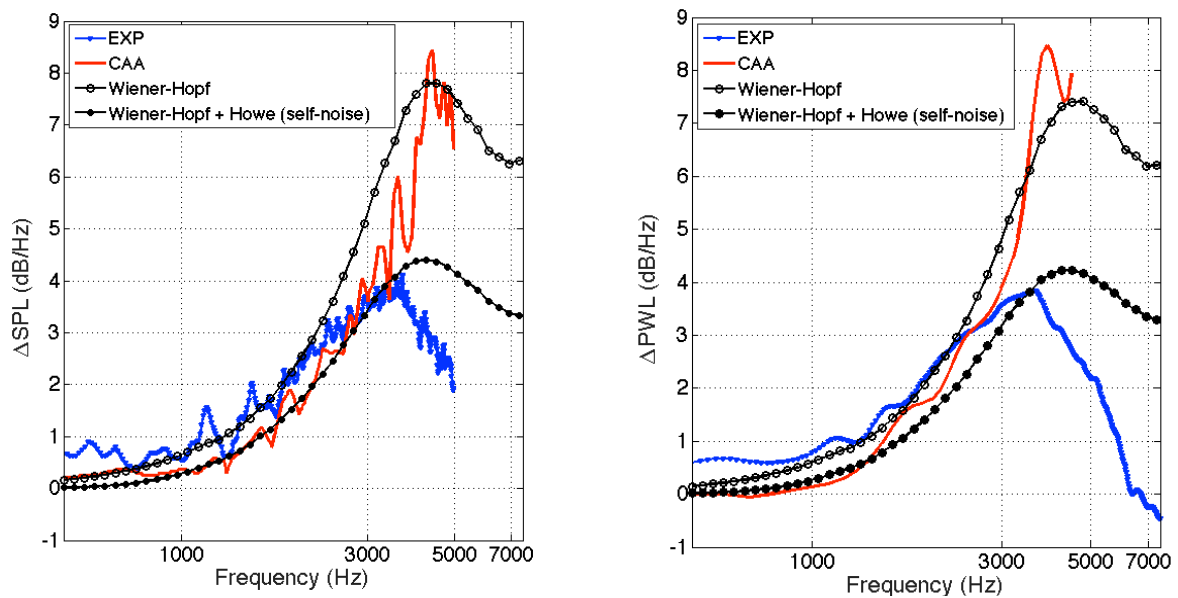

FIG. 26. Delta SPL spectra at $\theta=90^{\circ}$ observer (left) and delta PWL spectra (right) from CAA solutions ${ }^{2}$, WH analytical model (with and without the self-noise contribution), and experiment ${ }^{4}$.

\section{Application to a low-noise OGV}

Finally, the WH model is applied to the TurboNoiseBB configuration, including both the baseline and low-noise OGVs. The OGVs are assumed to radiate noise to the far-field as isolated flat plates, i.e., airfoil thickness, duct propagation and cascade effects are neglected here. Firstly, uniform inflow conditions are imposed by setting radially-averaged turbulence statistics (see Fig. 6). This is convenient for the WH formulation that assumes a periodic LE along the span, such as the "GanzUniform" OGV design in Fig. 27. This OGV design takes into account two significant geometrical modifications discussed in Section IV: (1) limitation of serration amplitude and (2) local suppression of LE serrations near the hub. Radially-averaged turbulence statistics are used in the WH analytical model for the baseline case $\left(h_{s}=0\right)$, which is compared to numerical, experimental, and analytical predictions using Amiet's model at approach OP in Fig. 28. The PWL from the WH analytical model has been computed from the contribution of 44 single-vanes $(+10 \log (44) \mathrm{dB})$, which corresponds to the vane count of the OGV stage in the experimental set-up. This implies that LE noise radiates to the free-field in contrast 
with the PWL spectrum from the extended Amiet's model in the TinA2D code, which accounts for duct propagation from an annular cascade. ${ }^{33}$ Although the PWL spectrum is over-predicted by the WH method at low and mid-range frequencies, the spectral shape is well-predicted, and the levels tend to converge to the Amiet-based solution at high frequency. This is mainly due to the free-field radiation assumption in the WH formulation, which is known to be a high frequency approximation. Overall, this result is considered to be sufficiently accurate to assess the aeroacoustic response of isolated airfoils with LE serrations.
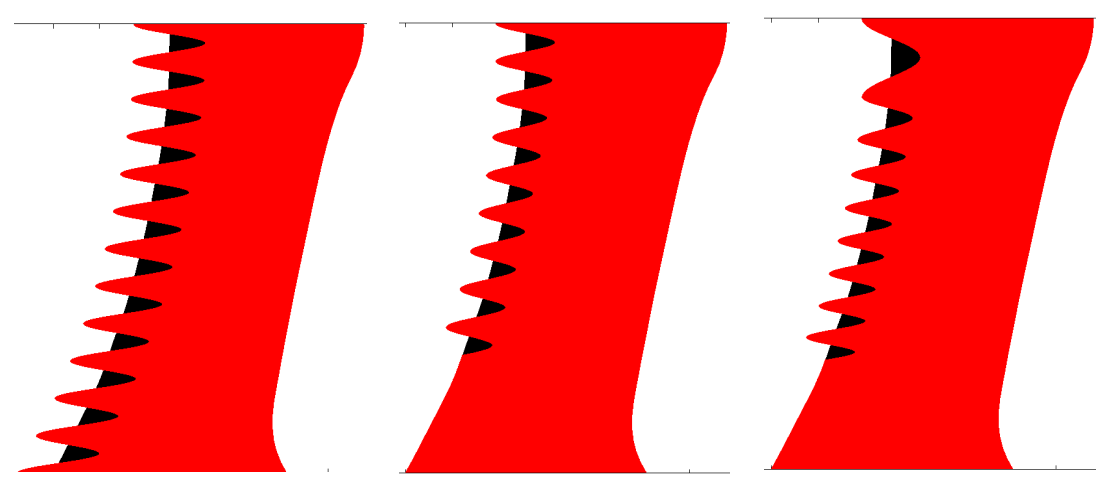

FIG. 27. 2D low-noise OGV 2D designs: "GanzUniform" (left), "GanzUniformOpt" (midle) and "GanzRadialOpt" (right)

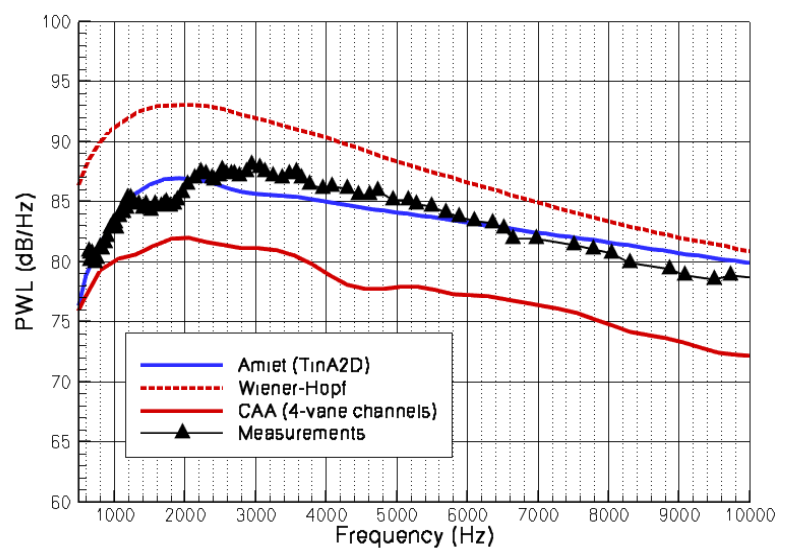

FIG. 28. PWL spectra at APP OP for the baseline OGV using different methods (CAA, WH, Amiet-based) and compared to measurements.

Then, the "GanzUniformOpt OGV design" is used to assess the acoustic performances at three different fan speeds that correspond to the acoustic certification OPs, using radially-averaged profiles of TI and TLS from RANS simulations provided by DLR (see Fig. 5) and mean flow values from Table I. The WH calculations are performed for a single vane, and the resulting PWL and delta PWL spectra are shown in Fig. 29. Significant PWL reductions can be observed at all OPs, although the design was optimized for the APP OP. This is consistent with the results shown in the delta PWL, where the maximum noise reduction is obtained at APP OP. It can be also observed that the frequency associated to the maximum PWL reduction increases with the mean flow speed (from APP to SDL). This trend is in good agreement with the basic design rules 
and initial results discussed in Section IV and provided in Table I, in which the maximum PWL reductions varies between 4 and $6 \mathrm{~dB}$. Moreover, the maximum PWL reduction from the WH model occurs at frequencies that are close to the maximum in the PWL spectra from experimental results, which have been highlighted with arrows. Although these maxima in the PWL spectra are not fully aligned with those from the Amiet-based predictions (a shift can be observed at approach OP), they agree quite well with those of the delta PWL spectra in Fig. 29 (top right). This suggests that the proposed low-noise OGV design might be effective to reduce broadband noise from the ACAT1 fan-OGV stage at various OPs.
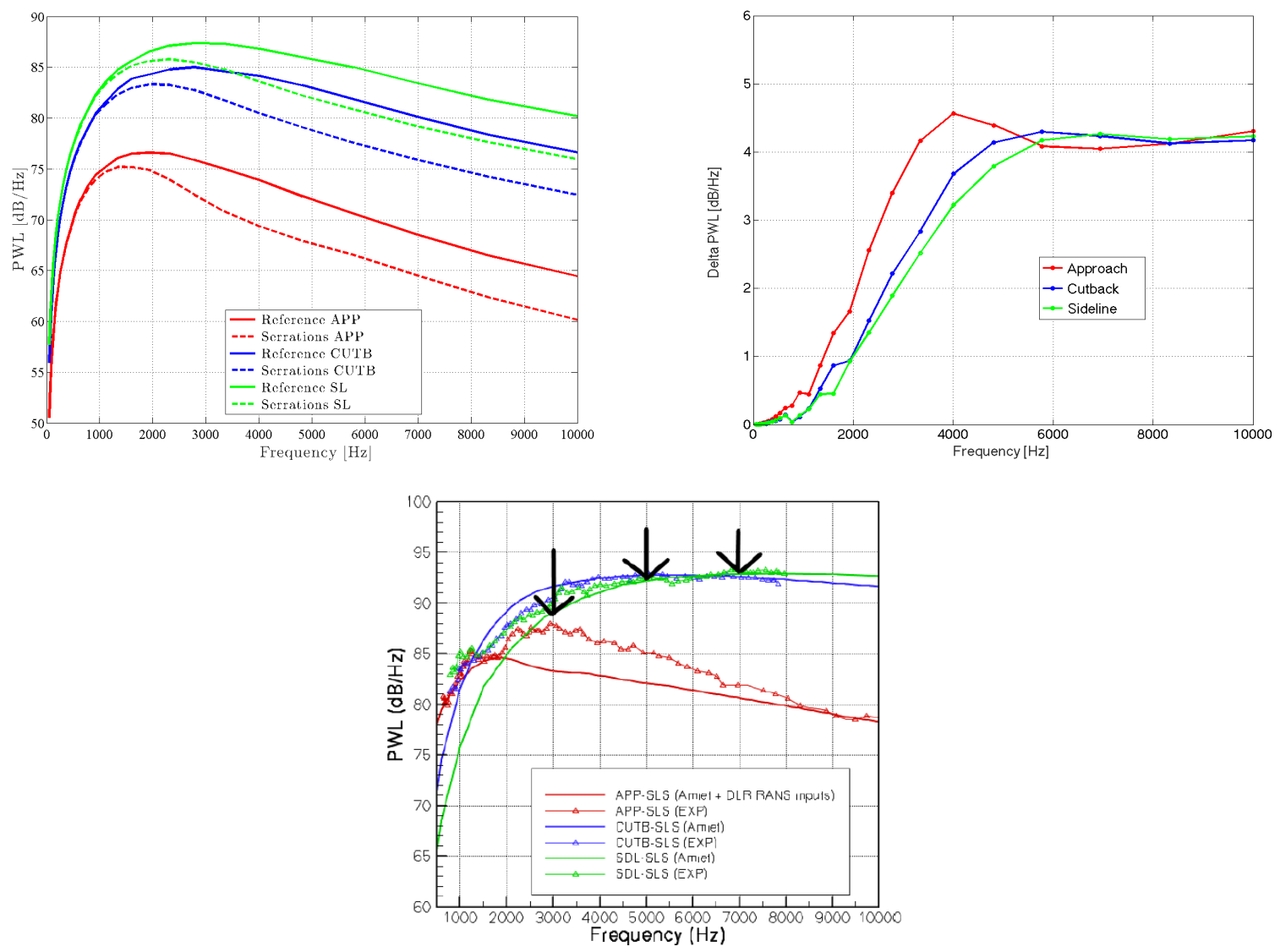

FIG. 29. PWL spectra (top left) and delta PWL spectra (top right) from WH calculations (1 vane) on reference and low-noise OGV designs at the 3 OPs, and PWL spectra from Amiet-based and experiment al results (bottom).

Finally, it is proposed here to split the actual "GanzRadialOpt" OGV in spanwise strips to account for the spanwise varying LE (see Fig. 27 on the right). To this end, 9 strips are defined along the span, where the last strip corresponds to the straight LE region near the hub, and the inflow characteristics are radially-averaged over each strip. The periodicity condition in the WH model is still valid for each strip, as the serration pattern is roughly preserved in the spanwise direction. Using these assumptions, the WH-based predictions (for a single vane) are presented in Fig. 30. It can be seen that the PWL spectrum provided by the free-field Amiet's solution ${ }^{28}$ for the baseline case (using the strip technique for the characteristics of the incoming flow) has been included in Fig. 30 (on the left). A good agreement can be observed between WH and Amiet 
solutions, which is consistent with the findings for the FLOCON test-case results in Fig. 24 (on the right). The shape of WHbased PWL spectra for the baseline and serrated LE vanes are quite similar to those shown in Fig. 29 (left, APP OP), but the strip technique leads to higher levels in the PWL spectrum. The analytical delta PWL spectrum is plotted in Fig. 30 (on the right), and compared to the most accurate CAA prediction from Fig. 22 (on the right). The WH prediction obtained from the strip technique is rather close to the one shown in Fig. 29 (with "GanzUniformOpt" design and radially-averaged profiles), but a slightly higher PWL reduction reaching $5 \mathrm{~dB}$ at approximately $3.5 \mathrm{kHz}$ can be observed. Although the WH analytical model predicts larger noise reductions than CAA simulations, the trends are relatively similar up to $7 \mathrm{kHz}$. The acoustic benefit predicted by the analytical model might be slightly over-estimated due to the flat plate and uniform mean flow assumptions. ${ }^{4-5}$ Then, it seems reasonable to conclude that the expected PWL reduction from the most promising low-noise OGV is within these two limits. The WH method provides an OAPWL reduction of $3.5 \mathrm{~dB}$ (2.3 dB from CAA), and a PWL reduction of at least $4 \mathrm{~dB}(2 \mathrm{~dB}$ from $\mathrm{CAA})$ can be achieved in the considered frequency range.
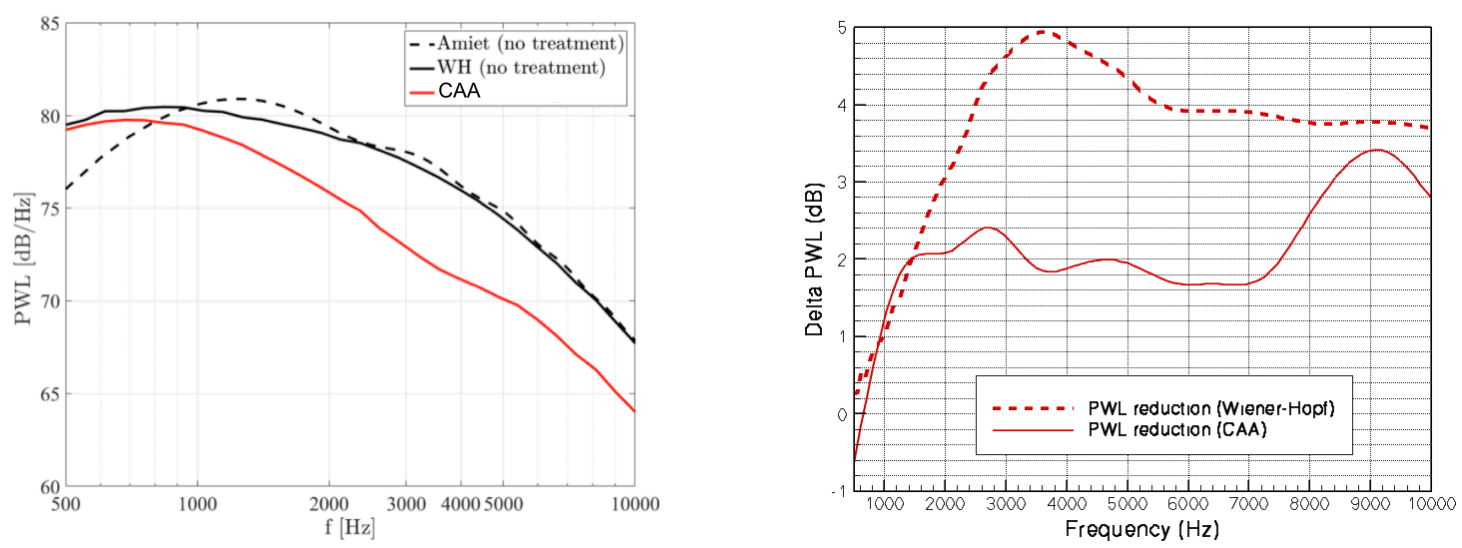

FIG. 30. PWL predictions (single vane calculation) from WH model for baseline and "GanzRadialOpt" OGVs and free-field Amiet model for the baseline case (left), and delta PWL prediction from the WH model compared to the CAA results (right).

\section{CONCLUSIONS AND PERSPECTIVES}

In this paper, the design of an optimized low-noise OGV with LE serrations has been performed by ONERA. To this end, sound power predictions using state-of-the-art CAA and analytical methods have been presented, including an evaluation of the aerodynamic performances from CFD simulations. The geometry of sinusoidal LE serration was adjusted using RANS-derived information and simple design rules from the literature. A preliminary estimation of acoustic performances using basic semi-empirical rules has shown that a PWL reduction of approximately 4-6 dB could be achieved on broadband noise using LE serrations. Optimized LE geometries have been obtained using spanwise-varying turbulent statistics (i.e., turbulence length scale) and industrial constraints suggested by Safran Aircraft Engines. The most promising 
low-noise OGV has been identified and down selected from RANS simulations using a mixing-plane approach to ensure reduced penalties on the loss coefficient and isentropic efficiency. The so-called "GanzRadialOpt" low-noise OGV including spanwise variations in the serration wavelength and amplitude has been investigated using a hybrid CAA method. Assumptions and limitations in the CAA simulations have been discussed, such as the use of a synthetic turbulence method that only models velocity disturbances that are perpendicular to the chord and the use of a filtered mean flow near the TE to avoid numerical instabilities. Additionally, an available analytical model for LE serrations has been used to confirm the PWL reductions from CAA predictions. This model, which is based on the Wiener-Hopf technique, has been extended here to account for airfoils with a finite span and three-dimensional far-field propagation.

It has been shown that the low-noise OGV can reduce the noise over a wide frequency range (from $1 \mathrm{kHz}$ to $10 \mathrm{kHz}$ ) with at least $2 \mathrm{~dB}$ around the maximum in the PWL spectrum in the bypass duct. An OAPWL attenuation of $2.3 \mathrm{~dB}$ then is expected in the broadband noise, according to the CAA simulations. To confirm this result, WH calculations have been presented and validated from the FLOCON turbulence-airfoil configuration, using available data from CAA simulations and measurements. The analytical method is shown to be quite accurate when applied to isolated airfoils with sinusoidal LE serrations. Then, the WH method has been applied to reproduce TurboNoiseBB configurations (with and without LE serrations), at three different flow speeds that are representative of the approach, cutback and sideline OPs. The PWL spectrum obtained at APP OP for the baseline OGV agrees well with the Amiet-based results and the experimental measurements, which makes this analytical model to be reliable for the investigated cases. Finally, acoustic performances of the "GanzRadialOpt" design shows a more significant PWL reduction of up to 4-5 dB with a spectral shape that is relatively close to that from the CAA simulations. The analytical delta OAPWL is equal to $3.5 \mathrm{~dB}$ which is consistent with the semiempirical and numerical values.

In summary, the assessment of acoustic performances provided by $3 \mathrm{D}$ CAA simulations and a complementary $2 \mathrm{D}$ analytical model is quite promising. The low-noise OGV design proposed by ONERA should allow for a noticeable OAPWL reduction while taking into account some industrial constraints. Next steps should be the manufacturing of a low-noise OGV and a test campaign in a dedicated rig facility (as scheduled in the framework of a starting new European project), in order to measure the acoustic benefit of such LE serrations with more realistic conditions.

\section{ACKNOWLEDGMENTS}

This project has received funding from the European Union's Horizon H2020 TurboNoiseBB program under grant agreement No 690714. ONERA would like to thank the DLR team (S. Guérin, C. Kissner, M. Behn, and S. Hakasson) for 
sharing their RANS data and for providing the required post-processed measurements from experimental measurements. Safran Aircraft Engines is also acknowledged for the technical support given.

\section{ABBREVIATIONS}

ACAT: AneCom AeroTest

ADP: $\quad$ Aerodynamic Design Point

APP: Approach

BBN: Broadband Noise

BPF: $\quad$ Blade Passing Frequency

CAA: Computational AeroAcoustics

CFD: Computational Fluid Dynamics

CFL: Courant-Friedrichs-Lewy

CPU: Central Processing Unit

CUTB: Cutback

FPR: $\quad$ Fan Pressure Ratio

FWH: $\quad$ Ffowes-Williams and Hawkings

LE: Leading Edge

LEE: Linearized Euler Equations

OAPWL: Overall Sound Power Level

OGV: $\quad$ Outlet Guide Vane

OP: $\quad$ Operating Point

PSD: $\quad$ Power Spectral Density

PWL: $\quad$ Power Level

SDL: $\quad$ Sideline

SPL: $\quad$ Sound Pressure Level

RANS: Reynolds Averaged Navier-Stokes

RMS: $\quad$ Root Mean Square

TE: $\quad$ Trailing Edge

TI: Turbulence Intensity

TLS: $\quad$ Turbulence Length Scale

WH: $\quad$ Wiener-Hopf

\section{APPENDIX A: MAIN STEPS TO DERIVE THE WIENER-HOPF ANALYTICAL MODEL}

This appendix presents the main steps to derive the WH model from Ayton and $\mathrm{Kim}^{8}$ and Lyu et al. ${ }^{34}$. The impinging gust $\widetilde{v_{l}}=\widetilde{v_{0}} e^{i(-\widetilde{\omega} t+\widetilde{\boldsymbol{k}} \cdot \widetilde{x})}$ interacts with a flat plate (described by a normalized function $F(\tilde{z}), \operatorname{such}$ as $\max (F)-\min (F)=$ $1 / 2$ ), which results in a pressure perturbation that is derived from a scattered velocity potential, 


$$
\tilde{p}=-\left(-\mathrm{i} \widetilde{k_{1}} \tilde{\phi}+\frac{\partial \widetilde{\phi}}{\partial \tilde{x}}\right)
$$

All variables are supposed to be harmonic and the acoustic velocity potential satisfies the Helmholtz equation (with $\left.1-M^{2}=\beta^{2}\right)$,

$$
\beta^{2} \frac{\partial^{2} \widetilde{\phi}}{\partial \tilde{x}^{2}}+\frac{\partial^{2} \widetilde{\phi}}{\partial \tilde{y}^{2}}+\frac{\partial^{2} \widetilde{\phi}}{\partial \tilde{z}^{2}}+2 \mathrm{i} \tilde{k} M \frac{\partial \widetilde{\phi}}{\partial \tilde{x}}+\tilde{k}^{2} \tilde{\phi}=0
$$

In order to satisfy the hard-wall boundary condition on the flat plate, it is necessary to ensure that,

$$
\forall \tilde{x}>\widetilde{h_{S}} F(\tilde{z}),\left.\frac{\partial \widetilde{\phi}}{\partial \tilde{y}}\right|_{\tilde{y}=0}=-\left.\widetilde{v_{l}}\right|_{\tilde{y}=0}
$$

Upstream of the flat plate LE the acoustic potential is supposed to be continuous,

$$
\forall \tilde{x}<\widetilde{h_{s}} F(\tilde{z}),[\tilde{\phi}]_{\tilde{y}=0^{-}}^{\tilde{y}=0^{+}}=0 .
$$

In the spanwise direction, a periodic boundary condition is applied,

$$
\left.\tilde{\phi}\right|_{\tilde{z}=1}=\left.\tilde{\phi}\right|_{\tilde{z}=0} \mathrm{e}^{i \widetilde{k_{3}}},\left.\frac{\partial \widetilde{\phi}}{\partial \tilde{z}}\right|_{\tilde{z}=1}=\left.\frac{\partial \widetilde{\phi}}{\partial \tilde{z}}\right|_{\tilde{z}=0} \mathrm{e}^{i \widetilde{k_{3}}}
$$

In order to solve the system of Eq. (A.2), the following steps are performed:

1. a convective transform: $h=\tilde{\phi} \mathrm{e}^{\mathrm{i} \widetilde{k_{1}} M^{2} \frac{\widetilde{x}}{\beta^{2}}}$

2. a change of variable: $\left\{\begin{array}{c}\xi=\frac{\tilde{x}}{\beta}-\frac{\widetilde{n_{s}}}{\beta} F(\tilde{z}) \\ \eta=\tilde{y} \\ \zeta=\tilde{z}\end{array}\right.$;

3. a Fourier transform in the $\xi$ variable: $H(\lambda, \eta, \zeta)=\int_{-\infty}^{+\infty} h(\xi, \eta, \zeta) \mathrm{e}^{\mathrm{i} \lambda \xi} \mathrm{d} \xi$.

The function $H$ can be obtained by using the separation variable technique: $H=Y(\lambda, \eta) Z(\lambda, \zeta)$, with separation constant $\chi$. Thus, Eq. (A.2a) becomes,

$$
\left\{\begin{array}{c}
Y^{\prime \prime}+\left(\left(\frac{\widetilde{\kappa_{1}}}{\beta} M\right)^{2}-\lambda^{2}-\chi^{2}\right) Y=0 \\
Z^{\prime \prime}+2 \mathrm{i} \lambda\left(\frac{\widetilde{h_{s}}}{\beta}\right) F^{\prime} Z^{\prime}+\left(\mathrm{i} \lambda \frac{\widetilde{h_{s}}}{\beta} F^{\prime \prime}-\lambda^{2}\left(\frac{\widetilde{h_{s}}}{\beta}\right)^{2} F^{\prime 2}+\chi^{2}\right) Z=0
\end{array} .\right.
$$

The system of Eq. (A.3) is solved using the boundary conditions. The solution for $H$ is given by, 


$$
\left\{\begin{array}{c}
H=\sum_{n=-\infty}^{+\infty} A_{n}(\lambda) \operatorname{sign}(\eta) e^{-|\eta| \sqrt{\lambda^{2}-\omega_{n}^{2}}} \mathrm{e}^{-\mathrm{i} \lambda \frac{\widetilde{h_{s}}}{\beta} F} e^{i \chi_{n} \zeta} \\
\chi_{n}=\widetilde{k_{3}}+2 n \pi \text { and } \omega_{n}=\sqrt{\left(\frac{\widetilde{k_{1}}}{\beta} M\right)^{2}-\chi_{n}^{2}}
\end{array}\right.
$$

The Wiener-Hopf technique is then used to obtain $A_{n}$ (more details are provided in Ref. ${ }^{34}$ ),

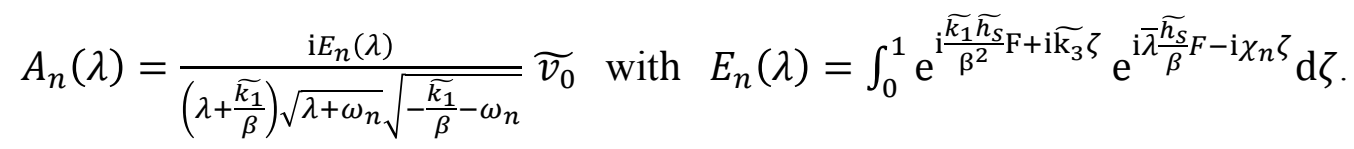

The function $h$ is finally recovered through an inverse Fourier transform, which is expressed in a stretched polar coordinate $\operatorname{system}\left(\tilde{r}=\sqrt{(\tilde{x} / \beta)^{2}+\tilde{y}^{2}}\right.$ and $\left.\tilde{y}=\tilde{r} \sin (\tilde{\theta})\right)$,

$$
h(\tilde{r}, \tilde{\theta}, \tilde{z})=\frac{1}{2 \mathrm{i} \pi} \int_{-\infty}^{+\infty} \mathrm{e}^{-\mathrm{i} \lambda\left(\tilde{r} \cos (\widetilde{\theta})-\frac{\widetilde{h_{s}}}{\beta} F\right)} H(\lambda, \tilde{r} \sin (\tilde{\theta}), \tilde{z}) d \lambda
$$

The integral in Eq. (A.6) is calculated by applying the steepest step descent method. Using Eq. (A.1), the final expression of the dimensionless pressure can be obtained in the far-field,

$$
\tilde{p}=\sum_{n=-\infty}^{n=+\infty} i\left(\frac{\widetilde{k_{1}}}{\beta^{2}}-\omega_{n} \cos (\widetilde{\theta})\right) \frac{e^{\mathrm{i} \pi / 4+\mathrm{i} \omega_{n} \widetilde{r}}}{\sqrt{\pi \tilde{r}}} \cos \left(\frac{\widetilde{\theta}}{2}\right) \frac{\mathrm{i} e^{\mathrm{i} \chi_{n} \tilde{z}} E_{n}\left(-\omega_{n} \cos (\widetilde{\theta})\right)}{-\omega_{n} \cos (\widetilde{\theta})+\frac{\widetilde{k_{1}}}{\beta}} \frac{e^{-\mathrm{i} \widetilde{k_{1}} M^{2} \frac{\widetilde{r} \cos (\widetilde{\theta})}{\beta}}}{\sqrt{-\frac{\kappa_{1}}{\beta}-\omega_{n}}} \widetilde{v_{0}}=L \widetilde{v_{0}}
$$

\section{APPENDIX B: 3D CORRECTION FOR APPLICATION TO FINITE SPAN AIRFOILS}

a) $b>>R_{o b s}$
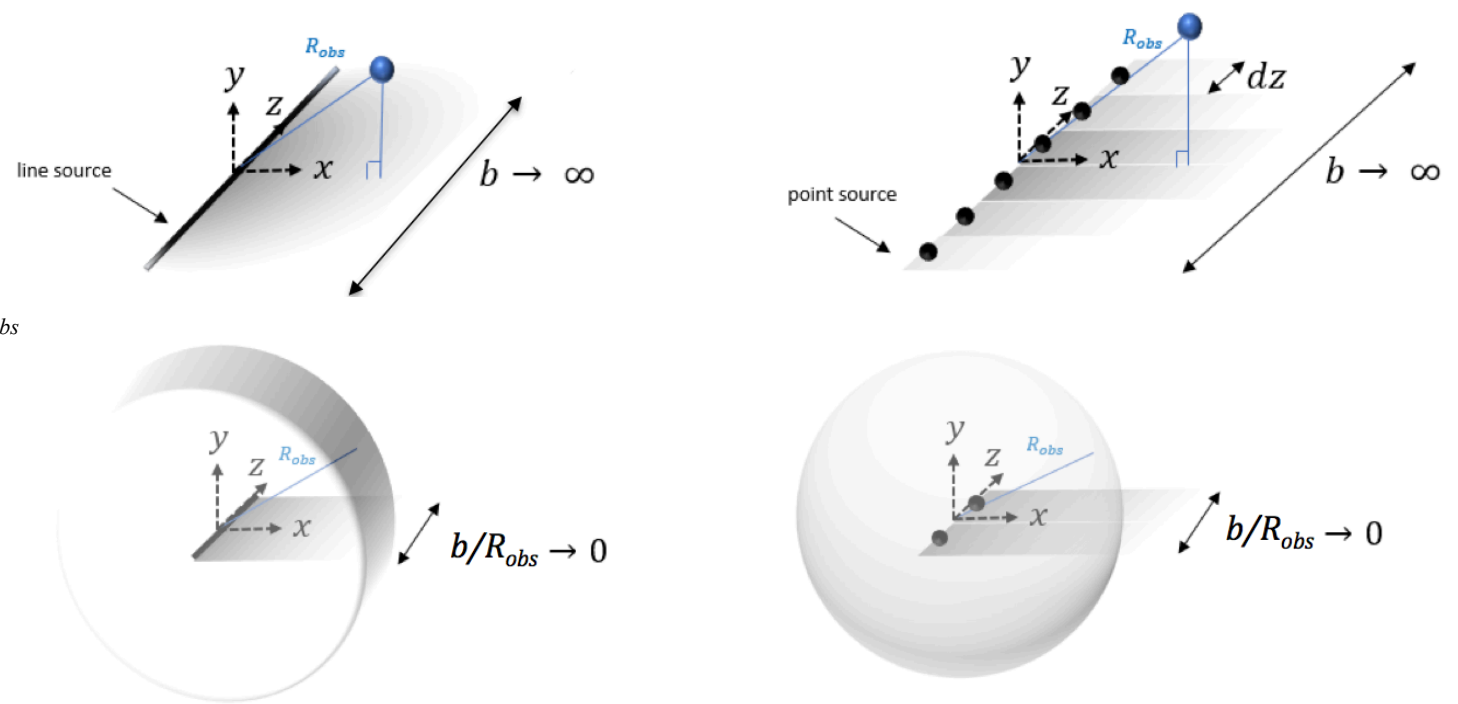

b) $b<<R_{\text {obs }}$

FIG. 31. Schematc of the different geometrical assumptions used to extend the WH formulation for finite span airfoils: line-source (left) and equivalent distributed point sources (right) for asymtotic limits $b>>R_{o b s}$ (top) and $b<<R_{o b s}$ (bottom) 
To account for finite span airfoils, a simple geometrical correction is applied in contrast with the reference model, which assumes an infinite span. Two asymptotic limits will be considered: $b \gg R_{o b s}$ and $b \ll R_{o b s}$ (with $R_{o b s}$ the distance between the observer and the center of the airfoil). For a line-source (Fig. 31, left), the 2D Green's function is proportional to the inverse of the square root of the distance to the observer, which leads to $p_{2 D}\left(R_{o b s}\right) \propto\left(A_{2 D} / \sqrt{R_{o b s}}\right) \exp \left(\mathrm{i}\left(\omega t-k R_{o b s}\right)\right)$. Let us now decompose the span in a number $n=b / d z$ (with $d z$ a small interval of the span) of point sources (Fig. 31, top right).

For each point source, $p_{3 D}(D) \propto\left(A_{3 D} / D\right) \exp (i(\omega t-k D))$. The 3D Green's function is proportional to the inverse of the distance $D$, where $D^{2}=R_{o b s}^{2}+z^{2}$ and $z$ corresponds to a location in the spanwise direction.

The two formulations should give the same intensity when an airfoil of an infinite span is considered, which leads to (using the Riemann sum approximation),

$$
\begin{gathered}
\left|p_{2 D}\left(R_{o b s}\right)\right|^{2}=\lim _{n \rightarrow \infty, d z \rightarrow 0} \sum_{i=1}^{n}\left|p_{3 D}\left(d z, D_{i}\right)\right|^{2}=2 \int_{0}^{\infty} \frac{\lim _{b \rightarrow \infty}\left(\left|A_{3 D}\left(b, R_{o b s}\right)\right|^{2}\right)}{\left(R_{o b s}^{2}+z^{2}\right)} \mathrm{d} z \\
\Rightarrow \lim _{b \rightarrow \infty}\left(\left|A_{3 D}\left(b, R_{o b s}\right)\right|^{2}\right)=\frac{1}{\pi}\left|A_{2 D}\right|^{2}
\end{gathered}
$$

When $b \ll R_{\text {obs }}$, the line-source tends to become compact. Let us consider two point sources infinitely close to each other from the observer point of view (Fig. 31, bottom right), and which are equivalent to a line-source. In order to keep a constant total radiated energy, one should verify,

$$
\begin{gathered}
\lim _{\underset{R_{o b s}}{\text { ob }} \rightarrow 0}\left(\frac{1}{b}\left(b 2 \pi R_{o b s}\right) \frac{\left|A_{2 D}\right|^{2}}{R_{o b s}}\right)=\lim _{\frac{b}{R_{o b s}} \rightarrow 0}\left(\frac{1}{2}\left(4 \pi R_{o b s}^{2}\right) \frac{\left|A_{3 D}\left(b, R_{o b s}\right)\right|^{2}}{R_{o b s}^{2}}\right) \\
\Rightarrow \lim _{\frac{b}{R_{o b s}} \rightarrow 0}\left(\left|A_{3 D}\left(b, R_{o b s}\right)\right|^{2}\right)=\left|A_{2 D}\right|^{2}
\end{gathered}
$$

Finally, for an airfoil with a finite span $b$, the integration in Eq. (B.1) is performed only between $-b / 2$, and $b / 2$,

$$
\left|p_{3 D}\left(b, R_{o b s}\right)\right|^{2}=2 \int_{0}^{\frac{b}{2}} \frac{\left|A_{3 D}\left(b, R_{o b s}\right)\right|^{2}}{\left(R_{o b s}^{2}+z^{2}\right)} d z=2\left|A_{3 D}\left(b, R_{o b s}\right)\right|^{2} \frac{\tan ^{-1}\left(\frac{b}{2 R_{o b s}}\right)}{R_{o b s}}
$$

In the case $b \gg R_{o b s}$ (with $R_{o b s}$ fixed), Eq. (B.3) becomes,

$\lim _{b \rightarrow \infty}\left(\left|p_{3 D}\left(b, R_{o b s}\right)\right|^{2}\right)=\lim _{b \rightarrow \infty}\left(2\left|A_{3 D}\left(b, R_{o b s}\right)\right|^{2} \frac{\tan ^{-1}\left(\frac{b}{2 R_{o b s}}\right)}{R_{o b s}}\right)=\frac{2}{\pi}\left|A_{2 D}\right|^{2} \frac{\frac{\pi}{2}}{R_{o b s}}=\left|p_{2 D}\left(R_{o b s}\right)\right|^{2}$

Thus, the reference formulation for an infinite span is recovered. 
When $b \ll R_{\text {obs }}$, using a Taylor expansion, one can show that,

$$
\left|p_{3 D}\left(b, R_{o b s}\right)\right|^{2} \approx\left|A_{2 D}\right|^{2} \frac{b}{R_{o b s}^{2}} .
$$

Eq. (B.5) relates the pressure radiated by an airfoil with a finite span through,

$$
\left|p_{3 D}\left(b, R_{o b s}\right)\right|^{2} \approx \frac{b}{R_{o b s}}\left|p_{2 D}\left(R_{o b s}\right)\right|^{2} .
$$




\section{REFERENCES}

${ }^{1}$ Polacsek, C., Reboul, G., Clair, V., Le Garrec, T., Deniau, H., Turbulence-Airfoil Interaction Noise Reduction Using Wavy Leading Edge: An Experimental and Numerical Study, Proceedings of Internoise, Noise Control Engineering of Japan (INCE/J) and Acoustical Society of Japan (ASI), Osaka (Japan), 2011.

${ }^{2}$ Clair, V., Polacsek, C., Le Garrec, T., Reboul, G., Gruber, M. and Joseph, P., Experimental and Numerical Investigation of Turbulence-Airfoil Noise Reduction Using Wavy Edges, AIAA Journal, Vol. 51, No. 11, 2013.

${ }^{3}$ Roger, M., Shram, C., De Santana, L., Reduction of Airfoil Turbulence-Impingement Noise by Means of Leading-Edge Serrations and/or Porous Materials, 19th AIAA/CEAS Aeroacoustics Conference, AIAA paper 2013-2108, 2013.

${ }^{4}$ Narayanan, S., Chaitanya, P., Haeri, S., Joseph, P., Kim, J.W., and Polacsek, C., Airfoil Noise Reductions Through Leading Edge Serrations, Physics of Fluids, vol. 27 No. 2, 2015.

${ }^{5}$ Chaitanya, P., Joseph, P., Vanderwel, C., Turner, J., Kim, J.W., and Ganapathisubramani, B., Performance and Mechanism of Sinusoidal Leading Edge Serrations for the Reduction of Turbulence-Aerofoil Interaction Noise, J. Fluid Mech., vol. 818, pp. 435-464, 2017.

${ }^{6}$ Gea-Aguilera, F., Gill, J., Angland, D., Zhang, X., Wavy Leading Edge Airfoils Interacting with Anisotropic Turbulence, 23rd AIAA/CEAS Aeroacoustics Conference, Denver (USA), 2017.

${ }^{7}$ Reboul, G., Cader, A., Polacsek, C., Le Garrec, T., Barrier, R., and Ben Nasr, N., CAA Prediction of Rotor-Stator Interaction Using Synthetic Turbulence: Application to a Low-Noise Serrated OGV, 23rd AIAA/CEAS Aeroacoustics Conference 2017, Denver (USA), 2017.

${ }^{8}$ Ayton, L.J. and Kim, J.W., An Analytical Solution for the Noise Generated by Gust-Airfoil Interaction for Plates With Serrated Leading Edges, J. Fluid Mech., vol. 853, pp. 513-536, 2018.

${ }^{9}$ Casalino, D., Avallone, F., Gonzalez-Martino, I., Ragni, D., Aeroacoustic Study of a Wavy Stator Leading Edge in a Realistic Fan/OGV Stage, J. Sound Vib., vol. 442, pp. 138-154, 2019.

${ }^{10}$ Polacsek, C., Cader, A., Barrier, R., De Laborderie, H., Gea-Aguilera, F., Aeroacoustic Design and Broadband Noise Predictions of a Turbofan Stage with Serrated Outlet Guide Vanes, ICSV26, Montreal (Canada), July 2019.

${ }^{11}$ Enghardt, L., The EU FP7 Research Project FLOCON - Objectives and First Results, Internoise 2010, Lisbon (Portugal), 2010.

${ }^{12}$ Hersh, A. S., Soderman, P. T., and Hayden, R. E., Investigation of Acoustic Effects of Leading-Edge Serrations on Airfoils, Journal of Aircraft, Vol. 11, No. 4, pp. 197-202, 1974.

${ }^{13}$ Hansen, K. L., Kelso, R. M., and Dally, B. B., Performance Variations of Leading-Edge Tubercles for Distinct Airfoil Profiles, AIAA Journal, Vol. 49, No. 1, pp. 185-194, 2011.

${ }^{14}$ Feinermann, J., Koushik, S., and Schmitz, F. H., Effect of Leading-Edge Serrations on Helicopter Blade-Vortex Interaction Noise, Proceedings of the 67th AHS International Annual Forum, Vol. 1, Curran Associates, Inc., Red Hook, NY, p. 25, 2011.

${ }^{15}$ Peake, N. and Parry, A.B., Modern Challenges Facing Turbomachinery Aeroacoustics, Annual Review of Fluid Mechanics, 44(1):227-248, 2012

${ }^{16}$ Tapken, U., Bauers, R., Neuhaus, L., Humphreys, N., Wilson, A., Stöhr, C., and Beutke, M., A New Modular Fan Rig Noise Test and Radial Mode Detection Capability, 17th AIAA/CEAS Aeroacoustics Conference, AIAA paper 2011-2897, Portland (USA), 2011. 
${ }^{17}$ Cader, A., Polacsek, C., Le Garrec, T., Barrier, R., Benjamin, F., and Jacob, M.C., Numerical Prediction of RotorStator Interaction Noise Using 3D CAA With Synthetic Turbulence Injection, 24th AIAA/CEAS Aeroacoustics Conference, Atlanta (USA), 2018.

${ }^{18}$ Guérin, S., Kissner, C., Kajasa, B., Jaron, R., Behn, M., Hakansson, S., Pardowitz, B., Tapken, U., Meyer, R., Enghardt, L., Noise Prediction of the ACAT1 Fan With a RANS-Informed Analytical Method: Success and Challenge, 25th AIAA/CEAS Conference, Delft (Netherlands), 2019.

${ }^{19}$ Behn, M., Pardowitz, B., and Tapken, U., Separation of Tonal and Broadband Noise Components by Cyclostationary Analysis of the Modal Sound Field in a Low-Speed Fan Test Rig, fan2018, Darmstadt (Germany), 18-20 April, 2018.

${ }^{20}$ Tapken, U., Behn, M., Spitalny, M., and Pardowitz, B., Radial Mode Breakdown of the ACAT1 Fan Broadband Noise Generation in the Bypass Duct Using a Sparse Sensor Array, 25th AIAA/CEAS Aeroacoustics Conference, Delfs (The Netherlands), 20-24 May, 2019.

${ }^{21}$ Cambier, L., Heib, S., and Plot, S., The Onera elsA CFD Software: Input from Research and Feedback from Industry, Mechanics \& Industry, Vol. 14, p. 159-174, 2013.

${ }^{22}$ Kissner, C., Guérin, S., Seeler, P., Billson, M., Chaitanya, P., Larana, P. C., de Laborderie, H., Lefarth, K., Lewis, D., Villar, G. M., Nodé-Langlois, T., and Polacsek, C., ACAT1 Benchmark of RANS-informed Analytical Methods for Fan Noise Prediction: Part I - Influence of the RANS Simulation, 26th AIAA/CEAS Aeroacoustics Conference, Reno (USA), 15-19 June, 2020.

${ }^{23}$ Donzis, D. A., Sreenivasan, K. R., and Yeung, P., Scalar Dissipation Rate and Dissipative Anomaly in Isotropic Turbulence, Journal of Fluid Mechanics, Vol. 532, pp. 199-216, 2005.

${ }^{24}$ Pope, S. B., Turbulent Flows, Cambridge Univ. Press, Cambridge, England, U.K., 2000.

${ }^{25}$ Ganz, U. W., Joppa P. D., Patten T. J. and Scharpf D. F., Boeing 18-Inch Fan Rig Broadband Noise Test, NASA report, CR-1998-208704, 1998.

${ }^{26}$ Redonnet, S., Manoha, E., and Sagaut, P., Numerical Simulations of Propagation of Small Perturbations Interacting with Flows and Solid Bodies, Proceedings of 7th AIAA/CEAS Aeroacoustics Conference, 2001.

${ }^{27}$ Tam, C. K. W., Advances in Numerical Boundary Conditions for Computational Aeroacoustics, Journal of Computational Acoustics, Vol. 6, No. 4, pp. 377-402, 1998.

${ }^{28}$ Amiet, R. K., Acoustic Radiation from an Airfoil in Turbulent Stream, Journal of Sound and Vibration, Vol. 41, No. 4 , pp. 407-420, 1975.

${ }^{29}$ Polacsek, C., Clair, V., Le Garrec, T., Reboul, G., and Jacob., M., Numerical Predictions of Turbulence/CascadeInteraction Noise Using Computational Aeroacoustics with a Stochastic Model, AIAA Journal, Vol. 53, No. 12, pp. 3551$3566,2015$.

${ }^{30}$ Atassi, H., Ali, A., Atassi, O., and Vinogradov, I., Scattering of Incident Disturbances by an Annular Cascade in a Swirling Flow, Journal of Fluid Mechanics, Vol. 499, pp. 111-138, 2004.

${ }^{31}$ Hixon, R., Sescu, A., and Sawyer, S., Vortical Gust Boundary Condition for Realistic Rotor Wake/Stator Interaction Noise Prediction Using Computational Aeroacoustics, Journal of Sound and Vibration, Vol. 330, pp. 3801-3817, 2011.

${ }^{32}$ Goldstein, M.-E., Aeroacoustics, McGraw-Hill, New York, pp. 63-66, 1976.

${ }^{33}$ Reboul, G., Polacsek, C., Lewy, S., and Heib, S., Ducted-Fan Broadband Noise Simulations Using Unsteady or Averaged Data, Internoise 2008 Conference, Inst. of Acoustics/Chinese Academy of Science, China, 2008. 
${ }^{34}$ Lyu, B., Ayton, L.J. and Chaitanya, P., On the Acoustic Optimality of Leading-Edge Serration Profiles, J. Sound and Vib. (462), 2019.

${ }^{35}$ Gea-Aguilera, F., Gill, J., Zhang, X., Synthetic Turbulence Methods for Computational Aeroacoustic Simulations of Leading Edge Noise, Comput. Fluids (157), pp. 240-252, 2017.

${ }^{36}$ Howe, M. S., Noise Produced by a Sawtooth Trailing Edge, The Journal of the Acoustical Society of America, (90), pp. 482-487, 1991. 